\title{
Witnessing galaxy preprocessing in the local Universe: the case of a star-bursting group falling into Abell $1367^{\star}$
}

\author{
L. Cortese ${ }^{1,2, \star \star}$, G. Gavazzi ${ }^{1}$, A. Boselli ${ }^{2}$, P. Franzetti ${ }^{3}$, R. C. Kennicutt ${ }^{4}$, K. O’Neil ${ }^{5}$, and S. Sakai ${ }^{6}$ \\ 1 Universitá degli Studi di Milano-Bicocca, P.zza della Scienza 3, 20126 Milano, Italy \\ e-mail: luca.cortese@astro.cf.ac.uk \\ 2 Laboratoire d'Astrophysique de Marseille, BP8, Traverse du Siphon, 13376 Marseille, France \\ 3 IASF - INAF, Sezione di Milano, via E. Bassini 15, 20133 Milano, Italy \\ 4 Institute of Astronomy, University of Cambridge, Madingley Road, Cambridge, CB3 OHA, UK \\ 5 NRAO, PO Box 2, Green Bank, WV 24944, USA \\ ${ }^{6}$ Department of Astronomy, University of California, Los Angeles, CA, 90095-1562, USA
}

Received 17 January 2006 / Accepted 13 March 2006

ABSTRACT

\begin{abstract}
We present a multiwavelength analysis of a compact group of galaxies infalling at high speed into the dynamically young cluster Abell 1367. Peculiar morphologies and unusually high $\mathrm{H} \alpha$ emission are associated with two giant galaxies and at least ten dwarfs/extragalactic HII regions, making this group the region with the highest density of star formation activity ever observed in the local clusters. Moreover $\mathrm{H} \alpha$ imaging observations reveal extraordinary complex trails of ionized gas behind the galaxies, with projected lengths exceeding $150 \mathrm{kpc}$. These unique cometary trails mark the gaseous trajectory of galaxies, witnessing their dive into the hot cluster intergalactic medium. Under the combined action of tidal forces among group members and the ram-pressure by the cluster ambient medium, the group galaxies were fragmented and the ionized gas was blown out. The properties of this group suggest that environmental effects within infalling groups may have represented a preprocessing step of the galaxy evolution during the high redshift cluster assembly phase.
\end{abstract}

Key words. galaxies: evolution - galaxies: clusters: individual: Abell 1367 - galaxies: interactions - galaxies: intergalactic medium

\section{Introduction}

Cluster of galaxies are unique laboratories for studying the dependence of galaxy evolution on the local environment. In the last decades a plethora of observational evidence has been collected, showing that the cluster environment is capable of perturbing both the morphology and the star formation history of galaxies (i.e. Gunn \& Gott 1972; Dressler 1980; Moore et al. 1996; Poggianti et al. 2001; Gavazzi et al. 2002a; Boselli et al. 2005). Most of what is known about the evolution of galaxies in clusters derives from studies of cluster cores, where the environmental effects are more severe, both locally and at higher redshift (i.e. Cayatte et al. 1990; Smail et al. 1997; Bravo-Alfaro et al. 2000; Boselli et al. 2005). Galaxies, however, interact with the harsh environment well before reaching the center of a cluster (Kodama et al. 2001; Treu et al. 2003). Moreover, the galaxies likely belong to infalling groups of galaxies which, according to the hierarchical scenario of the formation of large scale structures, represent the building blocks of today rich clusters of

^ Based on observations collected at the European Southern Observatory (proposal 71B-0154B), at the Italian Telescopio Nazionale Galileo (TNG) operated on the island of La Palma by the Centro Galileo Galilei of the INAF (Istituto Nazionale di Astrofisica) at the Spanish Observatorio del Roque de los Muchachos of the Instituto de Astrofisica de Canarias, at the Observatoire de Haute Provence (OHP) (France), operated by the CNRS, and at the Loiano telescope belonging to the University of Bologna (Italy).

$\star \star$ Present address: School of Physics and Astronomy, Cardiff University, Cardiff CF24 3YB, UK. galaxies. Galaxy groups may therefore represent natural sites for a preprocessing stage in the evolution of cluster galaxies (Fujita 2004; Mihos 2004) through tidal interactions, otherwise ineffective in high velocity dispersion environments. This suggests that at least part of the morphological transformation of cluster galaxies took place in earlier epochs in very different conditions than the ones observed in today's clusters (Dressler 2004). Unfortunately, witnessing preprocessing in local Universe is a real challenge since the current merger rate is considerably lower than in the past (Gottlöber et al. 2001). Presently we can observe several clusters experiencing multiple merging (Gavazzi et al. 1999; Donnelly et al. 2001; Ferrari et al. 2003; Cortese et al. 2004), but the structures involved are sub-clusters with a mass $\sim 5 \times 10^{14} M_{\odot}$, considerably higher than the typical mass of a compact group $\sim 10^{13} M_{\odot}$ (Mulchaey 2000).

This is also the case for the Abell cluster $1367(z \sim 0.0216)$ which is currently forming at the intersection of two filaments in the Great Wall, as revealed by the recent dynamical study of Cortese et al. (2004). Abell 1367 is a unique example among local, dynamically young clusters since, in addition to massive evolved substructures, it is also experiencing the merging of a compact group directly infalling into its core (Cortese et al. 2004). This merger was independently discovered by IglesiasPáramo et al. (2002) and Sakai et al. (2002) during deep H $\alpha$ surveys of nearby clusters, representing the region with the highest density of star forming systems ever observed in the local Universe. We refer to this infalling group as the Blue Infalling Group (BIG), as named by Gavazzi et al. (2003b). BIG has a mean recessional velocity of $\langle V\rangle=8230 \mathrm{~km} \mathrm{~s}^{-1}$ and a dispersion 
of only $\sim 150 \mathrm{~km} \mathrm{~s}^{-1}$ (Gavazzi et al. 2003b). Sakai et al. (2002) argued that BIG lies in the cluster background, since it exceeds by $\sim 1700 \mathrm{~km} \mathrm{~s}^{-1}$ the mean cluster velocity of $\langle V\rangle=6484 \mathrm{~km} \mathrm{~s}^{-1}$ $\left(\sigma_{V}=891 \mathrm{~km} \mathrm{~s}^{-1}\right.$, Cortese et al. 2004). Contrary to this, the dynamical analysis presented by Cortese et al. (2004), is consistent with an infalling scenario, as also proposed by Gavazzi et al. (2003b). Moreover this picture of BIG as an infalling cluster is supported by X-ray observations - Sun \& Murray (2002), using Chandra observations, discovered extended gas features and a ridge near the SE cluster center. They proposed that these features are associated with a new merging component penetrating the SE sub-cluster. These observational evidences suggest that we are witnessing, to our knowledge for the first time in the local Universe, a compact group infalling into a core of a dynamically young cluster. Because of its proximity, it represents a unique laboratory, allowing us to study in great detail a physical process that should be typical of clusters at higher redshift. The study of this group could help us shed light on the role of preprocessing on the evolution of galaxies in today's rich clusters.

During the last few years we have collected a multiwavelength dataset aimed at reconstructing the evolutionary history of this unique group of galaxies. The observations and data reduction are presented in Sect. 2; the peculiar properties of this group are described in Sect. 3 and their origin is discussed in Sect. 4, including a comparison with the Stephan's Quintet. Finally in Sect. 5 we conclude with a possible evolutionary scenario for this group and we discuss the importance of infalling groups on the evolution of cluster galaxies at higher redshift. Throughout this paper we assume for Abell 1367 a distance modulus $\mu=34.84$ mag (Sakai et al. 2000), corresponding to $H_{0}=70 \mathrm{~km} \mathrm{~s}^{-1} \mathrm{Mpc}^{-1}$.

\section{Observations}

\subsection{HI observations}

Using the refurbished 305-m Arecibo Gregorian radio telescope we observed the BIG region at $21 \mathrm{~cm}$ in March 2005. We obtained observations in 4 positions covering the group center and its NW outskirt (see Fig. 4). Given the instrument's beam at $21 \mathrm{~cm}\left(3.5^{\prime} \times 3.1^{\prime}\right)$ the three central positions $(97-114,97-120$ and 97-125), unlike BIG-NW, are not independent. Data were taken with the $L$-Band Wide receiver, using nine-level sampling with two of the 2048 lag subcorrelators set to each polarization channel. All observations were taken using the positionswitching technique, with each blank sky (or OFF) position observed for the same duration, and over the same portion of the telescope dish (Az and $\mathrm{El})$ as the on-source $(\mathrm{ON})$ observation. Each $5 \mathrm{~min}+5 \mathrm{~min} \mathrm{ON}+\mathrm{OFF}$ pair was followed by a $10 \mathrm{~s}$ $\mathrm{ON}+\mathrm{OFF}$ observation of a well-calibrated noise diode. Three pairs of observations were obtained per position, reaching an rms noise of $\sim 0.2 \mathrm{mJy}$. The velocity resolution was $2.6 \mathrm{~km} \mathrm{~s}^{-1}$ and the pointing accuracy is about $15^{\prime \prime}$. Flux density calibration corrections are good to within $10 \%$ or better (O’Neil 2004).

Using standard IDL data reduction software available at Arecibo, corrections were applied for the variations in the gain and system temperature with zenith angle and azimuth. A baseline of order one to three was fitted to the data, excluding those velocity ranges with $\mathrm{HI}$ line emission or radio frequency interference (RFI). The velocities were corrected to the heliocentric system, using the optical convention, and the polarizations were averaged. All data were boxcar smoothed to a velocity resolution of $12.9 \mathrm{~km} \mathrm{~s}^{-1}$ for further analysis. The obtained spectra are given in Fig. 5.

\section{2. $\mathrm{H} \alpha$ imaging}

We observed BIG using the Device Optimized for the LOw RESolution (DOLORES) attached at the Nasmyth B focus of the $3.6 \mathrm{~m}$ TNG in the photometric nights of 17th May and 18th June, 2004. The observations were taken through a [SII] narrow band filter centered at $\sim 6724 \AA$ with a width of $\sim 57 \AA$ covering the red-shifted $\mathrm{H} \alpha$ and [NII] lines. The underlying continuum was taken through a broadband (Gunn) $r^{\prime}$ filter. Images were split in 6 exposures of $1200 \mathrm{~s}$ in the narrow band filter and 5 exposures of $300 \mathrm{~s}$ in the $r^{\prime}$ broadband filter, for a total of $2 \mathrm{~h}$ and $25 \mathrm{~min}$ exposure respectively. Seeing was $\sim 1.2$ arcsec. Photometric calibration was achieved by observing the spectrophotometric star Feige 34. After bias subtraction and flat-fielding, the images were combined. The intensity in the combined OFF-band frame was normalized to that of the combined ON-band one by the flux ratio of several field stars. The NET image was obtained by subtracting the normalized OFF-band frame to the ON-band one. $\mathrm{H} \alpha+[\mathrm{NII}]$ fluxes and $E W \mathrm{~s}$ are obtained as described in Boselli et al. (2002).

Ten additional $r^{\prime}$ exposures of $600 \mathrm{~s}$ each were taken in the photometric nights of 1st-4th February 2006 using the same telescope, instrumental set-up and with a mean seeing of $\sim 1.1$ arcsec. The resulting image, obtained from the combination of all the $r^{\prime}$ exposures, has a total exposure time of $125 \mathrm{~min}$, reaching a $1 \sigma$ depth of $\mu_{r^{\prime}}=27.4 \mathrm{mag} \operatorname{arcsec}^{-2}$. The $r^{\prime}$-band and NET frames are shown in Figs. 1 and 2 respectively.

\subsection{MOS spectroscopy}

We observed the BIG region in MOS mode with the ESO/3.6 m and with the TNG telescope. The ESO/3.6 m observations were taken in the photometric nights of May 5th and 6th 2003 with the ESO Faint Object Spectrograph and Camera (EFOSC). We used the MOS mode of EFOSC to obtain the spectra of 9 of the emitting line knots. The EFOSC spectrograph was used with a $300 \mathrm{~g} / \mathrm{mm}$ grating and the $2048 \times 2048$ thinned Loral CCD detector, which provided coverage of the spectral region 3860-8070 A. Slit widths of 1.75" yielded a resolution of $\sim 19 \AA$. We obtained eleven exposures of $1530 \mathrm{~s}$, for a total of $\sim 4.65 \mathrm{~h}$.

The TNG observations were taken in the photometric nights of 26th March and 22nd April 2004 with DOLORES. We used the MOS mode of DOLORES to obtain the spectra of 8 of the emitting line knots and the nuclear region of CGCG97-125 (Zwicky et al. 1961). The DOLORES spectrograph was used with a grating which provided coverage of the spectral region 3200-8000 ̊. Slit widths of $1.6^{\prime \prime}$ yielded a resolution of $\sim 17 \AA$. We obtained six exposures of $1800 \mathrm{~s}$, for a total of $3 \mathrm{~h}$. All the emitting line regions observed in MOS spectroscopy are shown in Fig. 9.

In addition we took long slit drift-scan spectra of the bright galaxies CGCG97-114 and CGCG97-125 using the Loiano/1.52 m and the OHP/1.93 m telescopes respectively. At Loiano, the BFOSC spectrograph was used with a $300 \mathrm{~g} / \mathrm{mm}$ grating and $1300 \times 1340$ thinned EEV CCD detector, which provided a spectral coverage $3600-8900 \AA$. A slit width of $2.00^{\prime \prime}$ yielded a resolution of $\sim 20 \AA$. At OHP, the CARELEC spectrograph was used with a $300 \mathrm{~g} / \mathrm{mm}$ grating and $2048 \times$ 1024 EEV CCD detector, which provided a spectral coverage 3400-7000 A. A slit width of 2.50" yielded a resolution of $\sim 10 \AA$. Both the observations were taken in the "drift-scan" mode, with the slit parallel to the galaxy major axis, drifting 


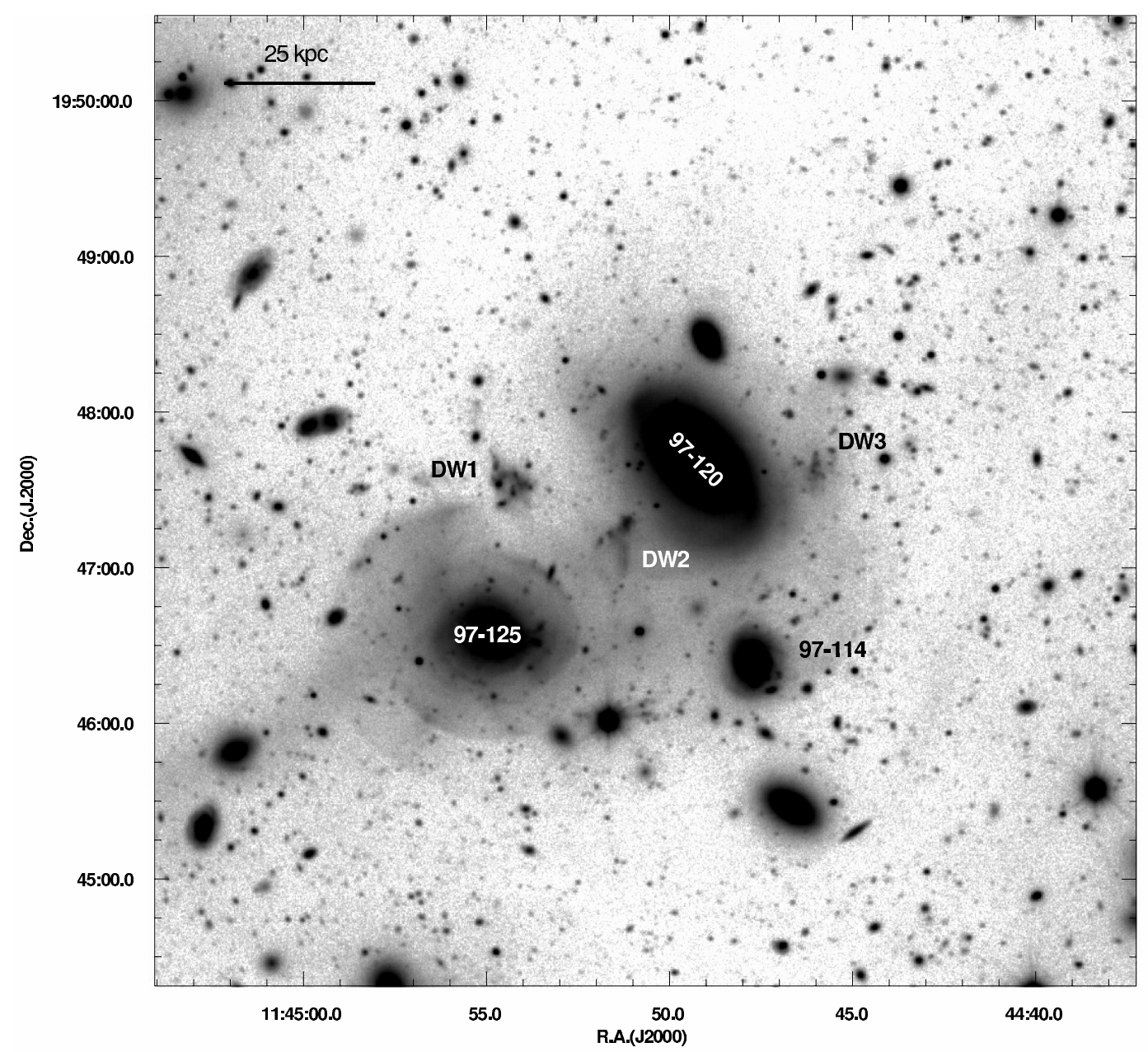

Fig. 1. Stellar shells are seen around galaxy CGCG97-125 in the $r^{\prime}$ band image of BIG. The three bright galaxies and the three dwarfs composing BIG are labeled.

over the optical surface of the galaxy. The total exposure time was $2400 \mathrm{~s}$ for CGCG97-114 and 1800 for CGCG97-125 respectively.

The reduction of all the spectra was carried out using standard tasks in the IRAF package. The $\lambda$ calibration was carried out using exposures of $\mathrm{He} / \mathrm{Ar}$ lamps for each slit. Typical errors on the dispersion solution are of $\sim 0.5-1 \AA$, as confirmed by the measurements of the sky lines. Spectra were flux-calibrated using the spectrophotometric standard stars: ltt 3864 for the ESO, Feige 67 for the TNG and Feige 34 for the Loiano and the OHP observations.

The redshift of each knot was derived as the mean of the individual redshift obtained from each emission line. Our results are shown in Table 1 and compared with the previous measurements by Sakai et al. (2002) and Gavazzi et al. (2003b). Due to the low resolution of our MOS $(R \sim 300)$, the mean redshift uncertainty is $\geq 150 \mathrm{~km} \mathrm{~s}^{-1}$, consistent with the discrepancies between the various entries in Table 1 . Therefore the present observations cannot be used for a detailed dynamical analysis of the group.

\subsubsection{Line measurements}

All spectra were shifted to the rest frame wavelength and normalized to their intensity in the interval 5400-5600 ̊. The fluxcalibrated, normalized spectra are presented in Fig. 13. Under visual inspection of the spectra we carried out the measurement of the emission lines. $\mathrm{H} \alpha(\lambda 6563)$ is bracketed by the weaker [NII] doublet ([NII1] $\lambda 6548$ and [NII2] $\lambda 6584$ ). The three lines are not well resolved, thus using the task SPLOT we performed a two Gaussian fit to the blended emissions providing an estimate of the line ratio [NII] $\lambda 6584 /(\mathrm{H} \alpha+[\mathrm{NII}] \lambda 6548)$. The two bright galaxies CGCG97-125 and CGCG97-114 show evidence for underlying Balmer absorption. We de-blended the underlying absorption from the emission lines as discussed in Gavazzi et al. (2004). From $\mathrm{H} \beta$ and $\mathrm{H} \alpha$ corrected for de-blending from [NII] we evaluate the Balmer decrement (assuming $T=10000 \mathrm{~K}$ and $\left.n=100 \mathrm{e} / \mathrm{cm}^{3}\right)$ :

$$
C 1=\frac{\log \left(\frac{1}{2.86} \times \frac{L_{\mathrm{H} \alpha}}{L_{\mathrm{H} \beta}}\right)}{0.33}
$$

(in the current notation: $A(\mathrm{H} \beta)=2.5^{*} C_{1}$ ).

The corrected line fluxes are derived, relative to $\mathrm{H} \beta$, using $C_{1}$ and the de-reddening law $f(\lambda)$ of Lequeux et al. (1979). When $\mathrm{H} \beta$ is undetected (only in the DW2b spectrum taken at ESO) we derive a $3 * \sigma$ lower limit to $C_{1}$ using (Gavazzi et al. 2004): $\mathrm{H} \beta<3 \times \operatorname{rms}_{(4500-4800)} \times \mathrm{H} \alpha H W H M$ assuming that $\mathrm{H} \alpha$ and $\mathrm{H} \beta$ have similar HWHM (Half Width Half Maximum).

In order to compare our observations with the ones presented by Sakai et al. (2002) we re-measured, using the method described above, the 1D spectra obtained from the $6.5 \mathrm{~m}$ MMT observations and kindly provided by these authors. The two sets of measurements presented in Table 2 are in fair agreement. 


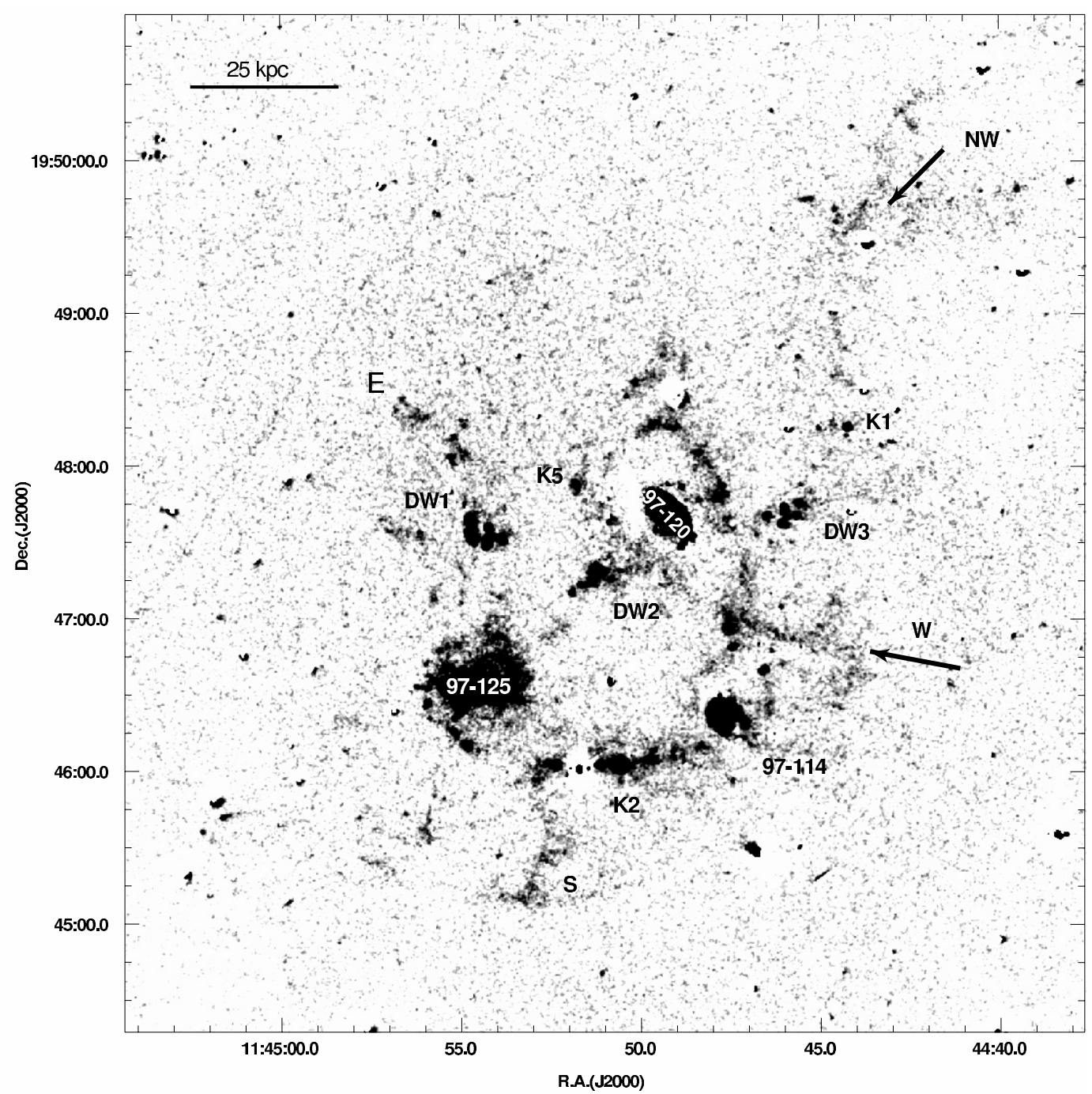

Fig. 2. Extended low surface brightness loops and trails (labeled NW, W and E) appear in the $\mathrm{H} \alpha+[\mathrm{NII}] \mathrm{NET}$ frame around the giant and dwarf galaxies of BIG.

Table 1. Redshifts of the galaxies in the BIG group.

\begin{tabular}{lrccccc}
\hline \hline Name & $\begin{array}{r}\text { RA } \\
(\mathrm{J} 2000)\end{array}$ & $\begin{array}{c}\text { Dec } \\
(\mathrm{J} 2000)\end{array}$ & \multicolumn{4}{c}{$\begin{array}{c}\text { Velocity } \\
\left(\mathrm{km} \mathrm{s}^{-1}\right)\end{array}$} \\
& & & TNG & ESO-MOS & Sakai02 & Gavazzi03 \\
\hline K1 & 114444.18 & 194816.0 & $8422 \pm 153$ & $8265 \pm 117$ & - & 8098 \\
DW3 d & 114445.97 & 194744.4 & - & $8564 \pm 151$ & - & - \\
DW3 e & 114445.97 & 194741.1 & - & $8072 \pm 124$ & - & - \\
DW3 a & 114446.43 & 194741.2 & $8490 \pm 180$ & - & 8266 & - \\
97-114b & 114446.56 & 194640.3 & - & $8656 \pm 132$ & 8504 & 8383 \\
$97-114 a$ & 114447.41 & 194649.8 & - & $8763 \pm 124$ & - & 8425 \\
K2 a & 114450.61 & 194605.1 & - & $8080 \pm 140$ & 8070 & 8089 \\
K2 b & 114449.71 & 194604.7 & $8309 \pm 165$ & - & - & - \\
DW2 c & 114451.12 & 194718.7 & $8380 \pm 188$ & - & - & - \\
DW2 b & 114451.17 & 194717.5 & - & $8221 \pm 146$ & - & 8077 \\
DW2 a & 114451.67 & 194713.5 & $8253 \pm 292$ & - & - & - \\
K5 & 114451.76 & 194752.7 & - & $8241 \pm 112$ & - & 7995 \\
DW1 b & 114453.78 & 194731.5 & - & - & 8070 & - \\
DW1 c & 114454.29 & 194728.6 & $8343 \pm 223$ & - & - & - \\
DW1 a & 114454.64 & 194732.9 & - & $8265 \pm 136$ & 8161 & 8067 \\
97-125b & 114454.89 & 194611.3 & $8261 \pm 191$ & $8396 \pm 132$ & 8170 & - \\
K3 & 114455.28 & 194803.3 & $8020 \pm 212$ & - & - & - \\
$97-125 a$ & 114455.99 & 194628.0 & - & - & - & 8330 \\
\hline
\end{tabular}


Table 2. Line fluxes, corrected for internal extinction, of the galaxies in the BIG group.

\begin{tabular}{lcccccccc}
\hline \hline Object & Tel. & C1 & {$[\mathrm{OII}]$} & $\mathrm{H} \beta$ & {$[\mathrm{OIII} 1]$} & {$[\mathrm{OIII} 2]$} & $\mathrm{H} \alpha$ & {$[\mathrm{NII} 2]$} \\
\hline K1 & ESO & - & $3.68 \dagger$ & 1.00 & $0.94 \dagger$ & $2.53 \dagger$ & - & - \\
K1 & TNG & 0.00 & 3.49 & 1.00 & 0.95 & 2.57 & 2.86 & 0.58 \\
DW3 a & TNG & 0.02 & 8.26 & 1.00 & 0.31 & 1.06 & 2.86 & 0.59 \\
DW3 d & ESO & 0.00 & 2.01 & 1.00 & 0.60 & 1.34 & 2.86 & 0.69 \\
97-114b & ESO & 0.33 & 4.25 & 1.00 & 0.57 & 1.83 & 2.86 & 0.26 \\
97-114a & ESO & 0.24 & 3.37 & 1.00 & 0.26 & 0.70 & 2.86 & 0.45 \\
CGCG97-114 & LOI & 0.75 & 2.61 & 1.00 & 0.21 & 0.36 & 2.86 & 0.64 \\
K2 a & ESO & 0.17 & 4.06 & 1.00 & 0.77 & 2.09 & 2.86 & 0.50 \\
K2 b & TNG & 0.23 & 8.43 & 1.00 & 0.64 & 0.82 & 2.86 & 0.32 \\
DW2 a & TNG & 0.16 & 5.45 & 1.00 & 0.73 & 0.99 & 2.86 & 0.60 \\
DW2 b & ESO & $>0.1$ & $>5.18$ & $1.00 \ddagger$ & $<1.00$ & $<0.99$ & 2.86 & $<0.61$ \\
K5 & ESO & 0.56 & - & 1.00 & 0.47 & 0.65 & 2.86 & 0.29 \\
DW1 b & MMT & 0.33 & 3.62 & 1.00 & 0.74 & 2.50 & 2.86 & 0.35 \\
DW1 c & TNG & 0.00 & 2.76 & 1.00 & 0.47 & 1.53 & 2.86 & 0.66 \\
DW1 a & ESO & 0.30 & - & 1.00 & 0.82 & 2.39 & 2.86 & 0.35 \\
DW1 a & MMT & 0.20 & 3.75 & 1.00 & 0.80 & 2.41 & 2.86 & 0.49 \\
97-125b & ESO & 0.55 & - & 1.00 & 0.51 & 1.25 & 2.86 & 0.37 \\
97-125b & TNG & 0.04 & 3.60 & 1.00 & 0.35 & 1.28 & 2.86 & 0.60 \\
CGCG97-125 & OHP & 0.88 & 9.23 & 1.00 & 1.06 & 1.97 & 2.86 & 0.84 \\
CGCG97-125 & TNG & 0.90 & 6.58 & 1.00 & 1.15 & 1.89 & 2.86 & 1.21 \\
K3 & TNG & 0.00 & 2.87 & 1.00 & 0.25 & 0.97 & 2.86 & 0.89 \\
\hline
\end{tabular}

$\dagger$ : Observed values (not corrected for internal extinction); $\ddagger$ : $\mathrm{H} \beta$ undetected.

\subsection{High resolution spectroscopy}

We obtained high dispersion long-slit spectra of CGCG97-125 and CGCG97-120 with the 1.93 m telescope of the Observatoire de Haute Provence (OHP), equipped with the CARELEC spectrograph coupled with a $2048 \times 512$ TK CCD, giving a spatial scale of 0.54 arcsec per pixel. The observations were carried out in the night of April 20, 2004 in approximately 2 arcsec seeing conditions through a slit of $5 \operatorname{arcmin} \times 2$ arcsec. The selected grism gives a spectral resolution of $33 \AA / \mathrm{mm}$ or $0.45 \AA /$ pix and $R \sim 5400$ in the region 6080-6990 $\AA$ containing the redshifted $\mathrm{H} \alpha(\lambda 6562.8 \AA)$, the $[\mathrm{NII}] \operatorname{doublet}(\lambda 6548.1,6583.4 \AA)$ and the [SII] doublet $(\lambda$ 6717.0, 6731.3 $\AA$ ).

\section{Results}

\subsection{The $\mathrm{H} \alpha$ trails}

The new $\mathrm{H} \alpha$ images of BIG obtained at the TNG reveal for the first time a spectacular $\mathrm{H} \alpha$ filamentary structure on top of which the star forming knots previously observed by Sakai et al. (2002) and by Gavazzi et al. (2003b) represent the tip of the iceberg (see Fig. 2). Multiple loops of ionized gas appear with a projected length exceeding $150 \mathrm{kpc}$ and a typical transverse size of $5 \mathrm{kpc}$, making these among the most extended low-brightness $\mathrm{H} \alpha$ emission features ever detected. One stream (labeled NW in Fig. 2) extends from the northern edge of the frame to the dwarf galaxy DW3, with an extension of $\sim 100 \mathrm{kpc}$. The second and brightest one (labeled W in Fig. 2) apparently traces a loop around the galaxy CGCG97-120 and seems connected to the bridge (labeled K2 in Fig. 2) between CGCG97-114 and CGCG97-125. If this is the case, the total projected extension of the NW and W trails would result $\sim 150 \mathrm{kpc}$. The looped trajectory traced by the trails in Fig. 2 should imagined as stretched along the line of sight, similar to a "bottle opener" that travels through a cork at a small angle $\theta$ with the line of sight. In this case, the projected length of the trails would be amplified by $\tan ^{-1} \theta$. In addition to the filamentary features, at least two other diffuse $\mathrm{H} \alpha$ regions (labeled $\mathrm{S}$ and $\mathrm{E}$ in Fig. 2) are detected.
The total diffuse $(\mathrm{H} \alpha+[\mathrm{NII}])$ emission (e.g. excluding the contribution of the three bright galaxies and of the ten dwarfs/HII regions previously discovered) results $\sim 1.2 \times 10^{-13} \mathrm{erg} \mathrm{cm}^{2} \mathrm{~s}^{-1}$, similar to the flux collected from CGCG97-125, and the typical surface brightness is $10^{-17.6}-10^{-18.3} \mathrm{erg} \mathrm{cm}^{-2} \mathrm{~s}^{-1} \operatorname{arcsec}^{-2}$.

The loop around CGCG97-120 alone contributes $\sim 2.4 \times$ $10^{-14}$ erg $\mathrm{cm}^{2} \mathrm{~s}^{-1}$, obtained integrating the $\mathrm{H} \alpha+[\mathrm{NII}]$ emission in a circular corona of $10 \mathrm{kpc}$ radius and an annulus of $5 \mathrm{kpc}$ centered on CGCG97-120. The derived line intensity is 2.05 Rayleigh $\left(1\right.$ Rayleigh $=10^{6} / 4 \pi$ photons $\mathrm{cm}^{-2} \mathrm{~s}^{-1} \mathrm{sr}^{-1}$ ), corresponding to an emission measure (EM) of $5.7 \mathrm{~cm}^{-6} \mathrm{pc}$. Assuming a torus geometry with a circular section of radius $\sim 5 \mathrm{kpc}$ and a filling factor of 1 , the resultant plasma density is $n_{\mathrm{e}} \sim 3.3 \times 10^{-2} \mathrm{~cm}^{-3}$ and the ionized column density is $N_{\mathrm{e}} \sim 5 \times 10^{20} \mathrm{~cm}^{-2}$ (the inferred densities would be higher if the gas is in clumps or filaments, which is likely). The emission measure in the NW trail is $\sim 1.3 \mathrm{~cm}^{-6}$ pc with a plasma density of $n_{\mathrm{e}} \sim 1.1 \mathrm{~cm}^{-3}$.

The trails' geometry is strongly suggestive of a Rosetta orbit, typically found with the tidal disruption of a satellite galaxy. Contrary to other known examples of tidal streams (i.e. Ibata et al. 2001; Shang et al. 1998; Wehner \& Gallagher 2005) the features observed herein show strong $\mathrm{H} \alpha$ emission, but it is difficult to determine whether or not the features are associated with a diffuse stellar component. The loop around CGCG97-120 lies in fact within the galaxy's optical radius, making impossible any estimate of the continuum emission associated with the $\mathrm{H} \alpha$ feature. A diffuse intra-group light, with typical surface brightness $\mu_{r^{\prime}}$ between 25.2 and 26.6 mag arcsec ${ }^{-2}$, is detected in the center of the group (but not around DW1) and in its western part (where the $\mathrm{W} \mathrm{H} \alpha$ trail starts), while no continuum emission above $\mu_{r^{\prime}} \sim 27.4 \mathrm{mag} \operatorname{arcsec}^{-2}$ is associated with the NW and $\mathrm{S} \mathrm{H} \alpha$ trails (Fig. 3), implying an extremely high $\mathrm{H} \alpha$ equivalent width $(E W(\mathrm{H} \alpha+[\mathrm{NII}]) \geq 100-150 \AA)$. It is also interesting to note that the one clear example of tidal stellar trail $\left(\mu_{r^{\prime}} \sim 26 \mathrm{mag} \operatorname{arcsec}^{-2}\right)$ detected in this region and extending from CGCG97-125 to south-east (see Fig. 3) does not show $\mathrm{H} \alpha$ emission. 


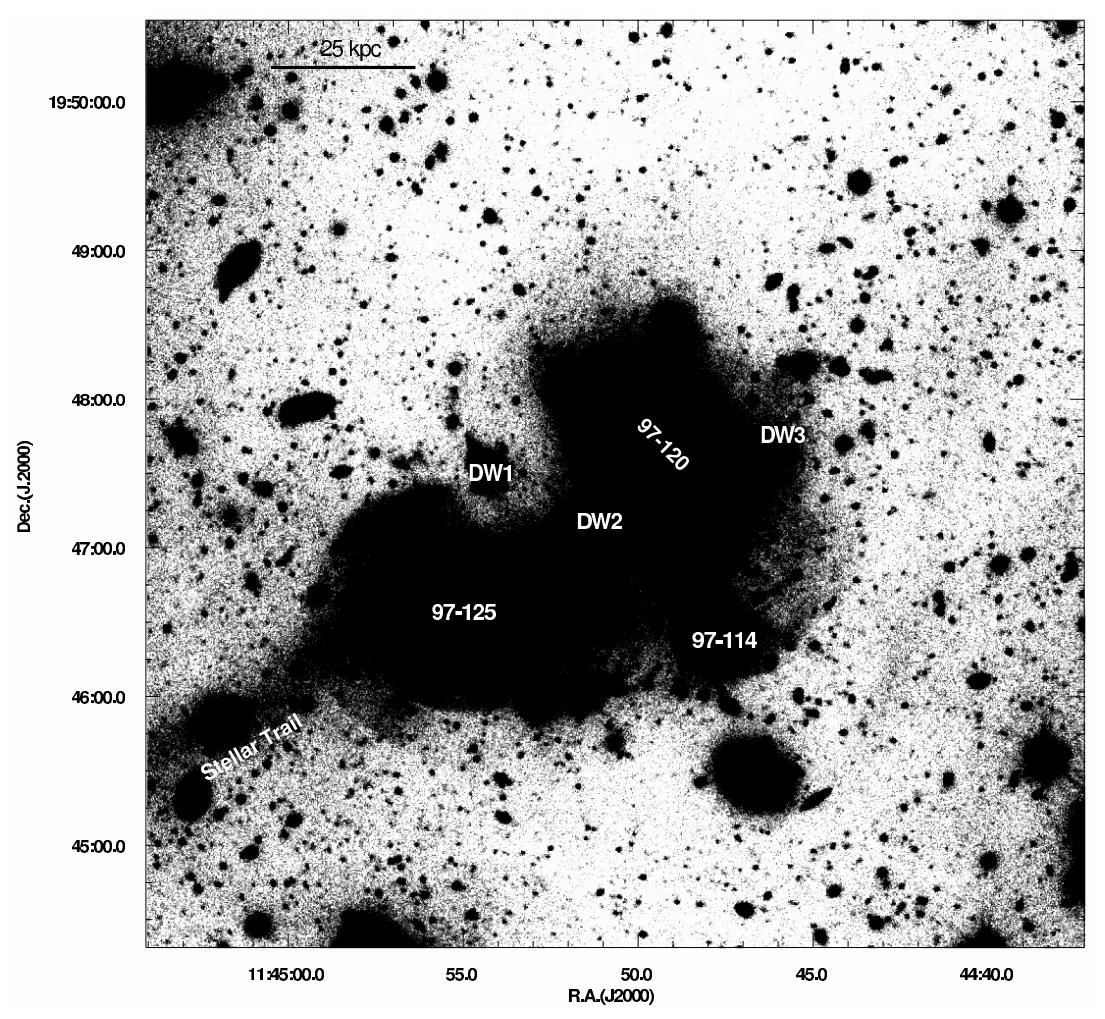

Fig. 3. A stellar trail starting from CGCG97-125 and diffuse intra-group light appear in the high contract $r^{\prime}$ band image of the BIG group.
Is there neutral gas associated with the $\mathrm{H} \alpha$ trails? The new HI observations obtained at Arecibo, combined with the higher resolution HI map previously available from Sakai et al. (2002), give additional hints on the properties of these extraordinary features. Figure 5 shows two HI spectra: one (solid line) obtained averaging three (non-independent) spectra from regions 97-125, 97-120, 97-114 (Fig. 4), the other (dotted line) obtained from the independent pointing BIG-NW. In the combined spectrum we detect $\sim 6.5 \times 10^{9} M_{\odot}$ in the two-horn peak at $8000<V<$ $9000 \mathrm{~km} \mathrm{~s}^{-1}$, consistent with $\sim 3.9 \times 10^{9} M_{\odot}$ from CGCG97-125, $\sim 3 \times 10^{8} M_{\odot}$ from CGCG97-114 and $\sim 2.2 \times 10^{9} M_{\odot}$ from a diffuse component, possibly associated with DW2 and K2, as determined by Sakai et al. (2002). However, in the same combined profile we detect a new component at $7500<V<8000 \mathrm{~km} \mathrm{~s}^{-1}$ with an HI mass $\sim 1 \times 10^{9} M_{\odot}$. In the same velocity interval, another $\sim 7 \times 10^{8} M_{\odot}\left(N_{\mathrm{HI}} \sim 3 \times 10^{20} \mathrm{~cm}^{-2}\right)$ component centered at $\sim 7870 \mathrm{~km} \mathrm{~s}^{-1}$ is detected in the independent BIG-NW region. In the velocity range $7500<V<8000 \mathrm{~km} \mathrm{~s}^{-1}$ there are no galaxies (see Table 1 and Fig. 9) in the observed beams (nor within their side-lobes) that could possibly contribute to this HI detection. The additional fact that this component, characterized by a width $\Delta V \sim 100 \mathrm{~km} \mathrm{~s}^{-1}$, in spite of being included in the datacube of Sakai et al. (2002), was not detected above their limiting sensitivity of $0.4 \mathrm{mJy} /$ beam implies that it extends over an area $\geq 1 \operatorname{arcmin}^{2}$. We argue that the new low-velocity HI component is associated with the diffused $\mathrm{H} \alpha$ trails, suggesting that the gaseous trails of BIG contain both neutral and ionized phases.

\subsection{The extragalactic star forming systems}

\subsubsection{Current star formation}

Contrary to the gaseous filaments, current star formation is clearly observed in all compact HII regions and dwarf and giant galaxies comprising BIG, suggesting that bursts of star formation are presently taking place within this group. At least ten

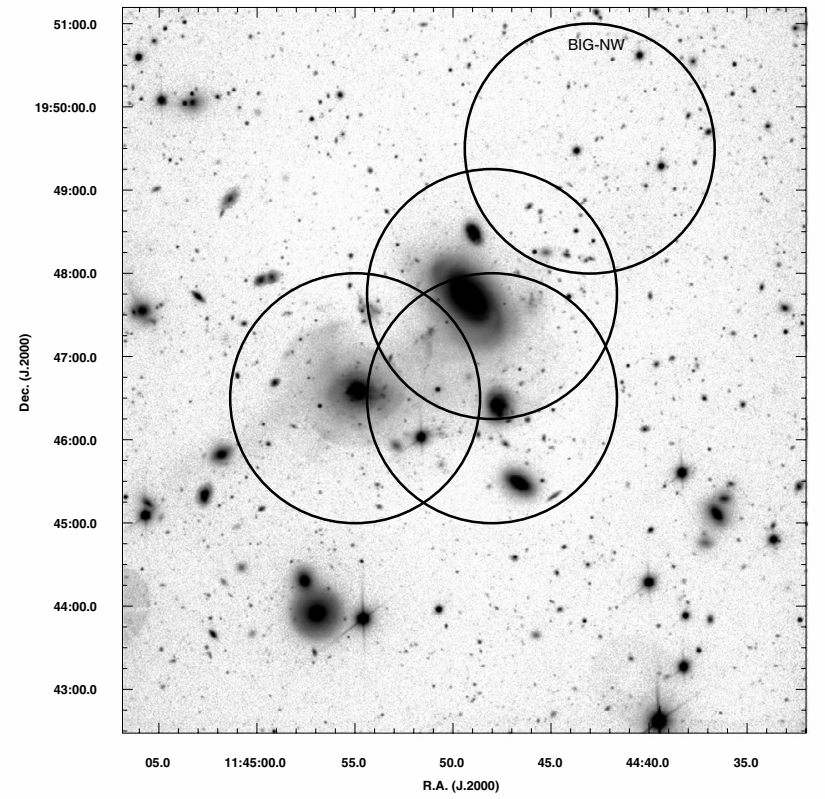

Fig. 4. The four Arecibo HI pointings obtained in the region of the BIG group, superposed to the $r^{\prime}$ band image. The size of each circle correspond to the telescope beam.

star-forming regions are associated with dwarf systems (or extragalactic HII regions) with $E W(\mathrm{H} \alpha+[\mathrm{NII}])$ often exceeding $100 \AA$ (see Table 3 ). These values are typical of HII regions (Kennicutt et al. 1989; Bresolin \& Kennicutt 1997) and usually observed in tidal dwarf candidates in interacting systems (Duc \& Mirabel 1998; Mendes de Oliveira et al. 2004). However, as remarked by Sakai et al. (2002), it is the first time that such a high density of star-forming galaxies has been seen in a nearby cluster, in spite of their having collected data over an area of 


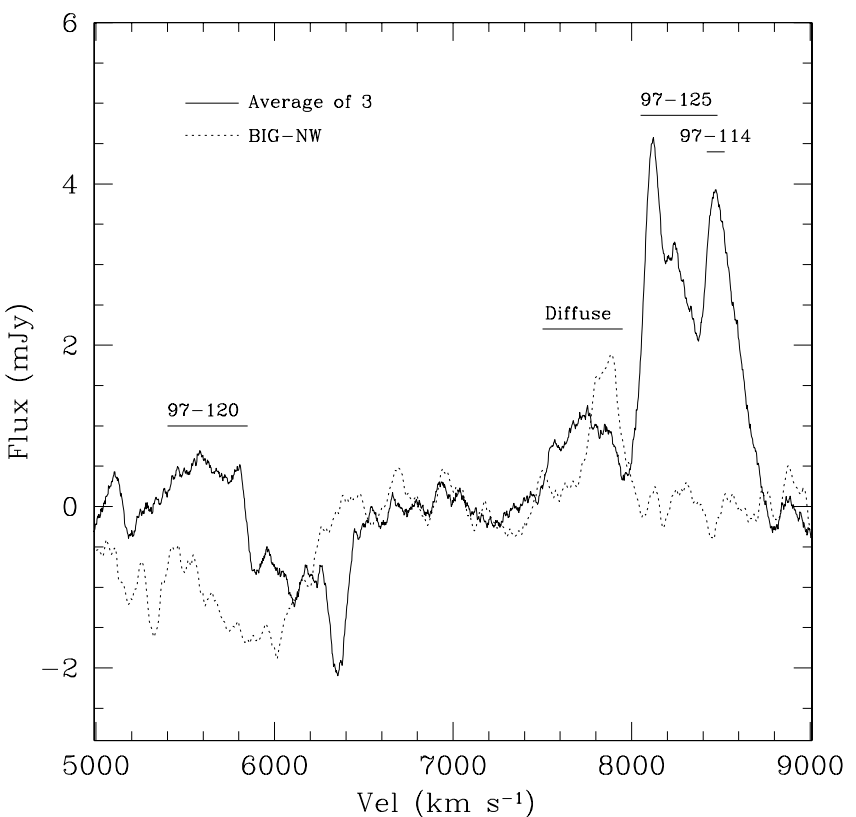

Fig. 5. Comparison between the combined HI spectrum obtained from the three Arecibo pointings (97-125, 97-120, 97-114; solid line) and the independent BIG-NW pointing (dotted line). The optical velocity width of the three bright Zwicky galaxies is given. The diffuse HI component is also indicated.

A1367, Coma, and the Virgo cluster approximately 500 times larger than the group size (Gavazzi et al. 1998, 2002b,c, 2006; Iglesias-Páramo et al. 2002; Boselli et al. 2002; Boselli \& Gavazzi 2002)

It is interesting to note that the dwarf galaxy DW2, and in particular the knot DW2c, shows clear Post-Starburst signatures in its spectrum, with low residual current star-formation, extremely blue continuum $(B-R \sim 0.16 \pm 0.20 \mathrm{mag})$, strong Balmer absorption $(E W(\mathrm{H} \delta) \sim 8 \AA)$ and [OII] and $\mathrm{H} \alpha$ in emission (see Fig. 13). These features suggest that the bulk of the starburst took place already $\leq 10^{8}$ years ago (e.g. Poggianti \& Barbaro 1997; Poggianti et al. 1999; Kauffmann et al. 2003).

\subsubsection{Kinematics}

Table 1 lists the positions and radial velocities of the objects measured spectroscopically (see also Fig. 9). Our observations confirm the physical association of all the emitting line objects within the bright galaxies CGCG97-114 and CGCG97-125 (Zwicky et al. 1961). The velocity of galaxies in BIG $(\langle V\rangle=$ $8230 \mathrm{~km} \mathrm{~s}^{-1}$ and $\sigma_{V}=170 \mathrm{~km} \mathrm{~s}^{-1}$ ) significantly exceeds the mean cluster velocity $\left(\langle V\rangle=6484 \mathrm{~km} \mathrm{~s}^{-1}, \sigma_{V}=891 \mathrm{~km} \mathrm{~s}^{-1}\right.$, Cortese et al. 2004), suggesting infall at $\sim 1700 \mathrm{~km} \mathrm{~s}^{-1}$ into the cluster core.

Although the MOS observations cannot be used for a detailed dynamical analysis of the group's members (due to their low resolution), they provide some information regarding the internal dynamic of DW3. DW3, in fact, presents remarkable kinematic peculiarities, as shown in Fig. 7, where the two emitting line knots DW3-d and DW3-e happened to lie within the same slit of one of our MOS observations taken at the ESO/3.6 m telescope. A relative velocity of $\sim 450 \mathrm{~km} \mathrm{~s}^{-1}$, unaffected by the uncertainty in the wavelength calibration ${ }^{1}$ is observed between the two knots. This high velocity gradient suggests that

1 We cannot however exclude that the different position of the two knots on the slit contributes to the observed velocity offset.

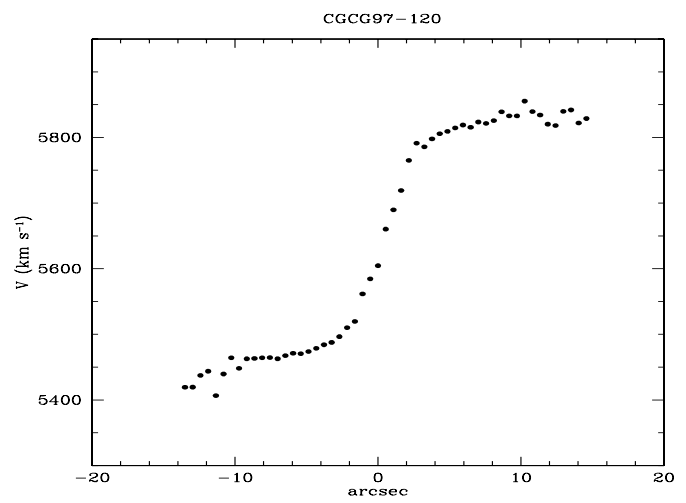

Fig. 6. The $\mathrm{H} \alpha$ rotation curve of CGCG97-120.

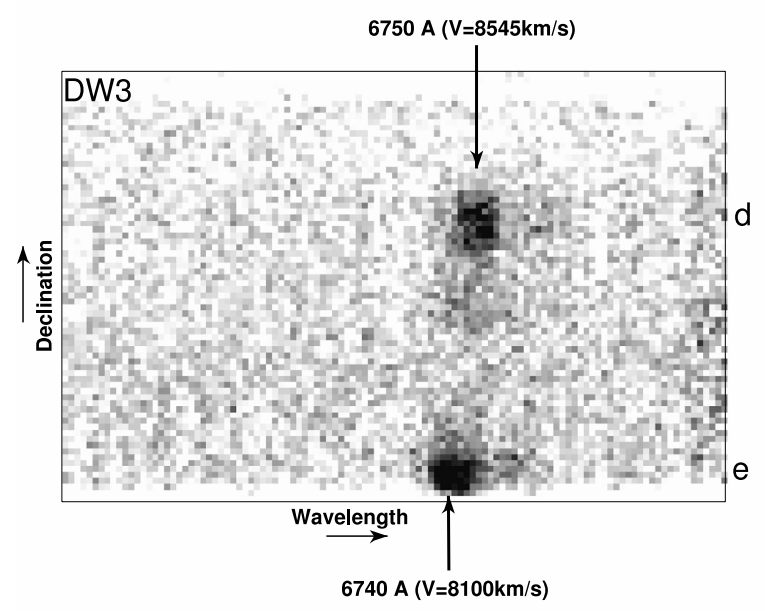

Fig. 7. The low resolution 2D spectrum obtained at ESO/3.6 for knots DW3d and DW3e, shows a significant difference $\left(\sim 500 \mathrm{~km} \mathrm{~s}^{-1}\right)$ in the their velocity.

the two knots do not form a gravitationally bound virialized system.

\subsubsection{Metal abundances}

In order to determine the metal content of BIG's constituents, we average five different empirical determinations based on the following line ratios: $R_{23} \equiv([\mathrm{OII}] \lambda 3727+[\mathrm{OIII}] \lambda 4959,5007) / \mathrm{H} \beta$

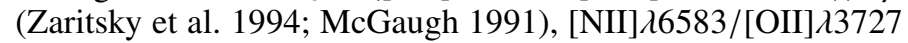
(Kewley \& Dopita 2002), [NII] $\lambda 6583 / \mathrm{H} \alpha$ (van Zee et al. 1998) and [OIII] $\lambda 5007 /[\mathrm{NII}] \lambda 6583$ (Dutil \& Roy 1999). Since the $R_{23}$ is not a monotonic function of the oxygen abundance, we used the line ratio [OIII] $\lambda 5007 /[\mathrm{NII}] \lambda 6583$ to distinguish between the lower and upper branches of the calibration, as suggest by Kobulnicky et al. (1999). The metallicities obtained from the various methods are shown individually and averaged in Table 4.

All the star-forming regions in BIG are metal-rich, with $8.5<12+\log (\mathrm{O} / \mathrm{H})<8.9$, and therefore extend the findings of Sakai et al. (2002).

It is well known that irregular and spiral galaxies follow a "metallicity - luminosity relation" (Skillman et al. 1989). Fig. 10 shows this relation for galaxies in the Virgo cluster (circles, obtained by Gavazzi et al. in prep. using the same methods and calibrations adopted here) and for the star-forming systems in BIG (triangles). The two brightest galaxies in the group (CGCG97-114 and CGCG97-125) have a normal metal content for their luminosity. Conversely the faintest $\left(L_{B}<10^{8} L_{\odot}\right)$ 
Table 3. Integrated $\mathrm{H} \alpha$ properties for some of the star forming systems in BIG.

\begin{tabular}{lcccc}
\hline \hline Name & $\begin{array}{c}r^{\prime} \\
\mathrm{mag}\end{array}$ & $\begin{array}{c}\mathrm{H} \alpha \text { flux } \\
\mathrm{erg} \mathrm{cm}^{-2} \mathrm{~s}^{-1}\end{array}$ & $\begin{array}{c}E W(\mathrm{H} \alpha+[\mathrm{NII}]) \\
\AA\end{array}$ & $\begin{array}{c}S^{\circ} R^{a} \\
M_{\odot} \mathrm{yr}^{-1}\end{array}$ \\
\hline 97125 & 13.99 & $(1.33 \pm 0.29) \times 10^{-13}$ & $27 \pm 3$ & 1.780 \\
97114 & 15.06 & $(6.59 \pm 0.71) \times 10^{-14}$ & $34 \pm 5$ & 0.880 \\
DW1 & 17.93 & $(1.60 \pm 0.17) \times 10^{-14}$ & $128 \pm 15$ & 0.113 \\
DW2 & 18.96 & $(3.67 \pm 0.86) \times 10^{-15}$ & $25 \pm 7$ & 0.023 \\
DW3 & 19.11 & $(4.47 \pm 0.96) \times 10^{-15}$ & $56 \pm 15$ & 0.027 \\
K1 & 21.46 & $(1.35 \pm 0.20) \times 10^{-15}$ & $330 \pm 85$ & 0.007 \\
K5 & 22.30 & $(1.15 \pm 0.39) \times 10^{-15}$ & $650 \pm 200$ & 0.014 \\
$97114 \mathrm{a}$ & 21.75 & $(1.14 \pm 0.22) \times 10^{-15}$ & $390 \pm 120$ & 0.008 \\
$97114 \mathrm{~b}$ & 22.00 & $(1.42 \pm 0.38) \times 10^{-15}$ & $680 \pm 90$ & 0.013 \\
$97125 \mathrm{~b}$ & 21.52 & $(1.90 \pm 0.20) \times 10^{-15}$ & $370 \pm 96$ & 0.014 \\
\hline
\end{tabular}

${ }^{a}$ Obtained assuming $S F R=L(\mathrm{H} \alpha) /\left(1.6 \times 10^{41}\right)($ Boselli et al. 2001), where $L(\mathrm{H} \alpha)$ is the $\mathrm{H} \alpha$ luminosity corrected for [NII] contribution and internal extinction using values obtained from spectroscopy (see Table 2 ).

Table 4. Metallicities $(12+\log (\mathrm{O} / \mathrm{H}))$ of the galaxies in the BIG group.

\begin{tabular}{lcccccccc}
\hline \hline Object & Tel. & \multicolumn{7}{c}{$12+\log (\mathrm{O} / \mathrm{H})$} \\
& & $R_{23}^{a}$ & $R_{23}^{b}$ & {$[\mathrm{NII}] /[\mathrm{OII}]^{c}$} & {$[\mathrm{NII}] / \mathrm{H} \alpha^{d}$} & {$[\mathrm{OIII}] /[\mathrm{NII}]^{e}$} & Mean & $\sigma$ \\
\hline K1 & TNG & 8.55 & 8.52 & 8.75 & 8.66 & 8.55 & 8.61 & 0.09 \\
DW3 a & TNG & - & 8.62 & - & 8.66 & 8.70 & 8.66 & 0.04 \\
DW3 d & ESO & 8.91 & 8.79 & 8.92 & 8.73 & 8.68 & 8.81 & 0.11 \\
97-114b & ESO & 8.59 & 8.52 & - & 8.30 & 8.48 & 8.47 & 0.13 \\
97-114a & ESO & 8.86 & 8.71 & 8.68 & 8.54 & 8.72 & 8.70 & 0.10 \\
CGCG97-114 & LOI & 9.00 & 8.83 & 8.85 & 8.70 & 8.92 & 8.86 & 0.11 \\
K2 a & ESO & 8.56 & 8.51 & 8.65 & 8.59 & 8.56 & 8.57 & 0.05 \\
K2 b & TNG & - & 8.64 & - & 8.39 & 8.64 & 8.56 & 0.14 \\
DW2 a & TNG & 8.53 & 8.44 & 8.61 & 8.67 & 8.71 & 8.59 & 0.11 \\
K5 & ESO & - & - & - & 8.35 & 8.66 & 8.50 & 0.22 \\
DW1 b & MMT & 8.56 & 8.53 & - & 8.43 & 8.48 & 8.50 & 0.06 \\
DW1 c & MMT & - & 8.42 & - & 8.64 & 8.58 & 8.55 & 0.12 \\
DW1 c & TNG & 8.81 & 8.70 & 8.84 & 8.71 & 8.66 & 8.75 & 0.08 \\
DW1 a & ESO & - & - & - & 8.43 & 8.49 & 8.46 & 0.04 \\
DW1 a & MMT & 8.55 & 8.52 & 8.67 & 8.58 & 8.53 & 8.57 & 0.06 \\
CGCG97-125 & OHP & - & 8.73 & - & 8.82 & 8.65 & 8.73 & 0.08 \\
CGCG97-125 & TNG & - & 8.49 & 8.77 & 8.98 & 8.72 & 8.74 & 0.20 \\
97-125b & ESO & - & - & - & 8.45 & 8.59 & 8.52 & 0.10 \\
97-125b & TNG & 8.76 & 8.64 & 8.75 & 8.67 & 8.67 & 8.69 & 0.05 \\
K3 & TNG & 8.89 & 8.75 & 8.90 & 8.84 & 8.78 & 8.83 & 0.07 \\
\hline
\end{tabular}

${ }^{a}$ Zaritsky et al. (1994); ${ }^{b}$ McGaugh (1991); ${ }^{c}$ Kewley \& Dopita (2002); ${ }^{d}$ Van Zee et al. (1998); ${ }^{e}$ Dutil \& Roy (1999).

star-forming knots show higher abundances than expected from their intrinsic luminosities. If these systems (K1, K5, K2, 97-114a, 97-114b and 97-125b) were isolated, independentlyevolved, dwarf galaxies, we would measure a metallicity 0.6-1.2 dex lower than observed. Moreover their abundances are consistent with the values measured for tidal dwarf systems $(12+\log (\mathrm{O} / \mathrm{H}) \sim 8.60$, independent from their absolute magnitude - e.g. Duc \& Mirabel 1999; Duc et al. 2000). At intermediate luminosities, the dwarf systems DW1, DW2 and DW3 have a high-metal content but are still consistent, within the calibration uncertainties, with the abundances observed in typical dwarf galaxies of their luminosity. However (as discussed in Sect. 3.2.2) we are not sure whether the star forming region DW3 should be considered as an individual galaxy or as an unbound collection of HII regions. In the latter case their metallicity would result $\sim 0.8$ dex higher than expected from the metallicity-luminosity relation.

As a result of the above considerations, we conclude that the HII regions in BIG are not normal, independently evolved dwarf galaxies, reinforcing the scenario proposed by Sakai et al. (2002) that these systems formed from enriched material stripped by tidal interactions from the two brightest galaxies in BIG.

\subsection{The brightest group member: CGCG97-125}

CGCG97-125 is classified as S0a (Gavazzi et al. 2003a), consistent with its red $B-R$ color index $(\sim 1.34 \mathrm{mag})$ and with the shape of the continuum optical spectrum (Fig. 11). However, this system is far from being a normal early type galaxy. The presence of stellar shells around CGCG97-125 (see Fig. 1) clearly indicates a past interaction/merger event. Numerical simulations predict that the stars from a satellite make a system of shells several $10^{8} \mathrm{yr}$ after the end of the merging event which lasts for more than 1 Gyr (Kojima \& Noguchi 1997).

This scenario is reinforced by the unusual dynamical state of the galaxy, as revealed by the high resolution spectra obtained at the OHP telescope. Velocity plots of CGCG97-125 were extracted from each spectrum by measuring the wavelength of the $\mathrm{H} \alpha$ line in each pixel along the slits. The three rotation curves so obtained are given in Fig. 8. In each diagram the recessional velocity is plotted as a function of position along the slit (the 

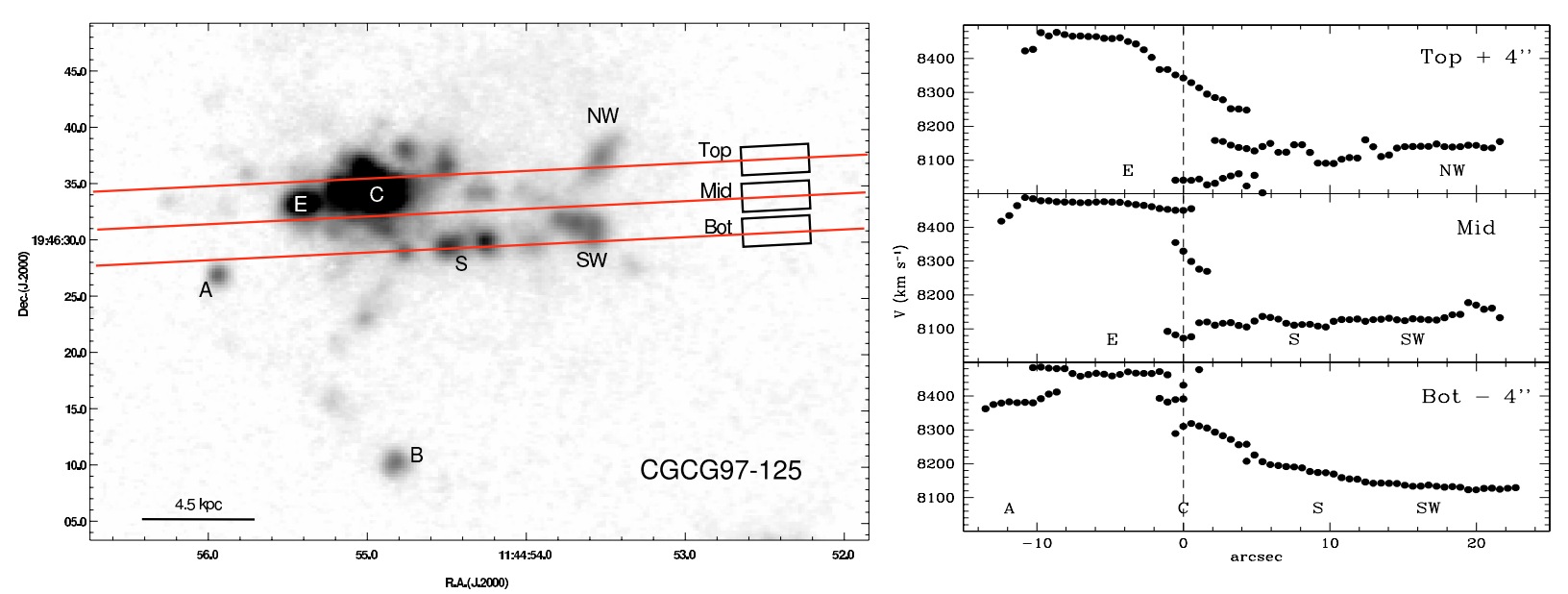

Fig. 8. Left panel: the position and the width (rectangular areas on the right) of the three slits obtained for CGCG97-125. The slits are superposed to the $\mathrm{H} \alpha+[\mathrm{NII}]$ net image. Right panel: the rotations curves obtained for the three indicated positions of CGCG97-125.

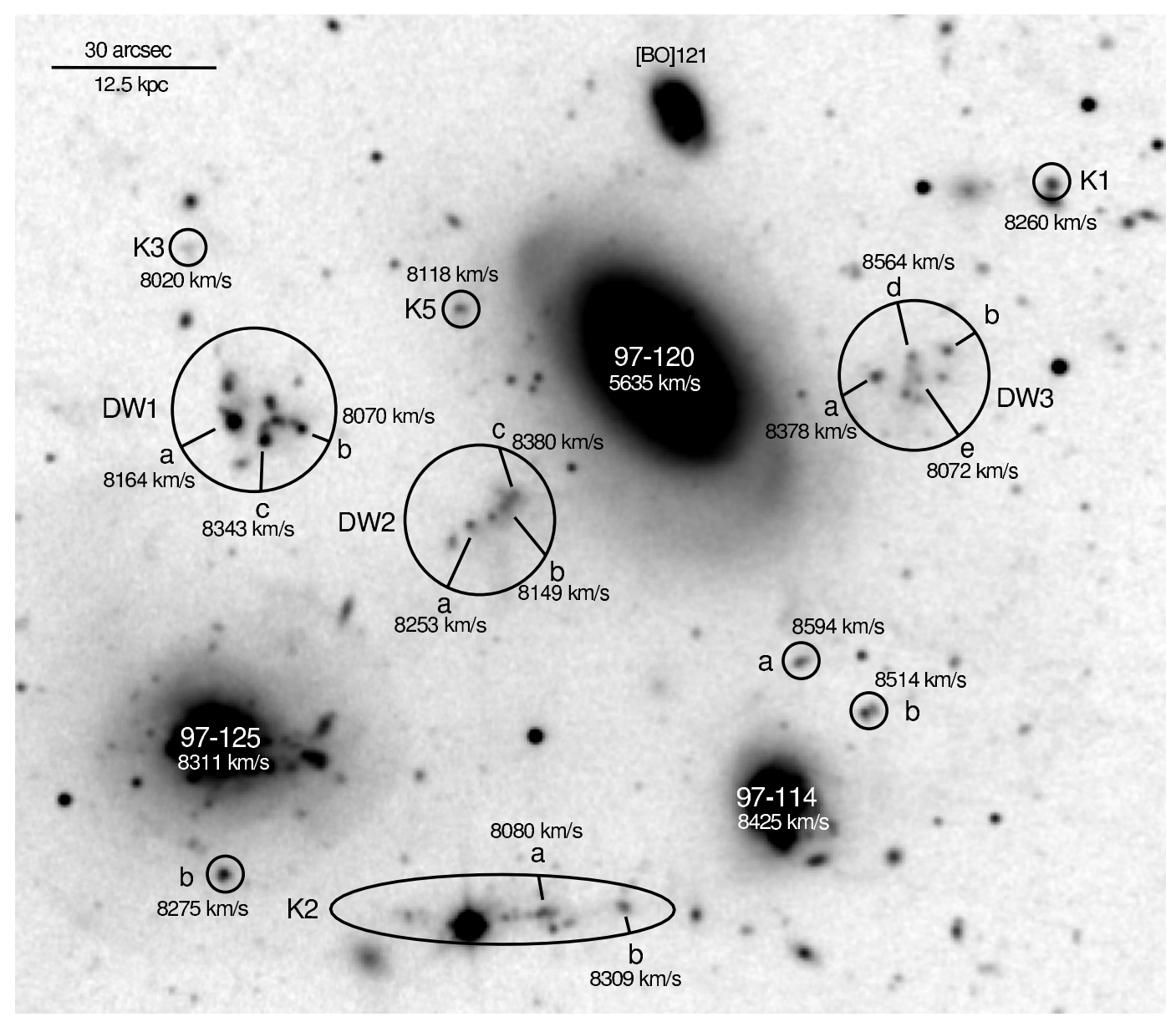

Fig. 9. High-contrast $\mathrm{H} \alpha+[\mathrm{NII}](\mathrm{ON})$ band frame of the BIG group. Labels indicate the star forming regions and their associated velocities.

spatial axis runs from $\mathrm{E}$ (left) to $\mathrm{W}$ (right)). All three spectra show multiple velocity components near the galaxy center $(\mathrm{C})$ where sudden velocity jumps of $\sim 100-150 \mathrm{~km} \mathrm{~s}^{-1}$ are detected. Even though examples of kinematic disturbances have been observed in normal galaxies (Rubin et al. 1999; Haynes et al. 2000) and interacting systems (Jore et al. 1996; Duc \& Mirabel 1998), features similar to those observed in CGCG97-125 were found only in UGC6697 (Gavazzi et al. 2001b), a merging systems in the NW region of Abell 1367.
Moreover, CGCG97-125 shows strong emission lines (Fig. 11) which are never seen in galaxies earlier than Sc (see e.g. Gavazzi et al. 2002a), indicating that this galaxy is still experiencing a strong burst of star formation, likely produced by the merging event - a kind of rejuvenated early type galaxy. If we compare the continua in the drift-scan integrated spectrum and the nuclear spectrum of CGCG97-125 we find that the first appears considerably bluer than the latter (see Fig. 11). Assuming that the nuclear continuum is representative of the old 


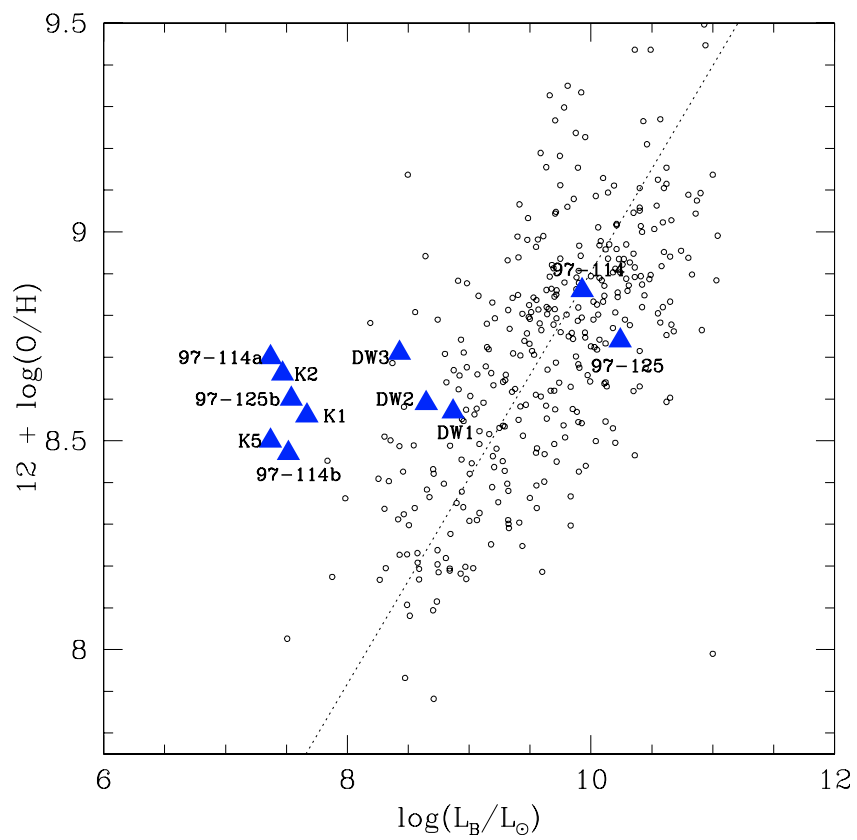

Fig. 10. The relation between metallicity and B-band luminosity (with linear best-fit) for galaxies in nearby clusters (empty circles, adapted from Gavazzi et al. 2004). The triangles mark the mean metallicity obtained for the star forming systems of BIG.

stellar population in this galaxy, pre-existing the burst, and that the drift-scan continuum is affected by the burst, we argue that the difference between the continua traces the recent star formation history of this galaxy, which can be therefore estimated. We use the SED fitting procedure proposed by Gavazzi et al. (2002a) and further developed by Franzetti (2005). We assume a star formation history (SFH) "a la Sandage":

$S F H(t, \tau)=\frac{t}{\tau^{2}} \times \exp \left(-\frac{t^{2}}{2 \tau^{2}}\right)$

and the Bruzual \& Charlot (2003, BC03) population synthesis models. We fit the nuclear spectrum of CGCG97-125, corrected for extinction ${ }^{2}$, assuming $t=13 \mathrm{Gyr}$, a Salpeter IMF ( $\alpha=2.35$ from 0.1 to $100 M_{\odot}$; Salpeter 1955), and exploring a parameter grid in metallicity $(Z)$, from $1 / 50$ to $2.5 Z_{\odot}$ in five steps: $0.0004,0.004,0.008,0.02$, and 0.05 . We also let $\tau$ to vary from 0.1 to $25 \mathrm{Gyr}$ in 45 approximately logarithmic steps. The best fit parameters obtained using the $\mathrm{BC} 03$ models are summarized in Table 5. The best value of $\tau$ is consistent with the one ( $\tau \leq 3.1$ Gyr) obtained by Gavazzi et al. (2002a) fitting a template of S0 galaxies. This result confirms that the continuum of the nuclear spectrum is dominated by an old stellar population of the same age expected for an unperturbed S0. By subtracting the best fit nuclear SED from the integrated SED of CGCG97-1253, we can estimate the starburst contribution to the galaxy emission, the burst age and mass. The best-fitting parameters obtained for the burst SED are summarized in Table 5. The burst age results $\sim 1.4-2.8 \mathrm{Gyr}$ and the stellar mass produced during

\footnotetext{
${ }^{2}$ We assume that the nuclear and integrated spectra are affected by the same amount of attenuation, as supported by the similar value of $C 1$ obtained in the two spectra (see Table 2). We thus estimate $A(\lambda)$ for the integrated SED using the ultraviolet spectral slope $\beta$ as discussed in Cortese et al. (2006) and assuming the Milky-Way attenuation curve (Boselli et al. 2003).

${ }^{3}$ Broad band integrated photometry of CGCG97-125 is taken from Cortese et al. (2005) and Gavazzi et al. (2003a).
}

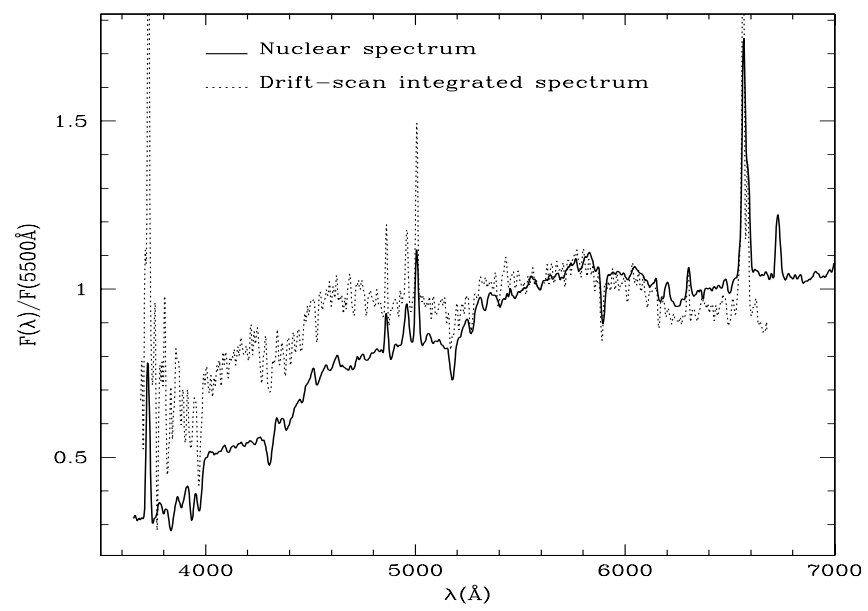

Fig. 11. Comparison between the drift-scan integrated (dotted) and nuclear (solid) spectrum of CGCG97-125.

Table 5. Best-fitting parameters for the nuclear and starburst component of CGCG97-125.

\begin{tabular}{lrccc}
\hline \hline Spectrum & $Z$ & Mass & $\tau$ & $t$ \\
& $\mathrm{Z}_{\odot}$ & $\log \left(M / M_{\odot}\right)$ & $\mathrm{Gyr}$ & $\mathrm{Gyr}$ \\
\hline Nuclear & 0.04 & 11.01 & $\leq 1.0$ & 13 \\
Starburst & 0.04 & 9.27 & $0.8-3.0$ & $1.4-2.8$ \\
\hline
\end{tabular}

the burst is $\sim 2 \times 10^{9} M_{\odot}$, consistent with the values previously obtained from independent estimates. The resulting best fit SED for CGCG97-125 is shown in Fig. 12 (solid line). We thus conclude that the star burst in CGCG97-125 was: initiated $\sim 1.5 \mathrm{Gyr}$ ago, probably produced by the minor merging of a $\sim 2 \times 10^{9} M_{\odot}$ satellite, and is still taking place. Our result points out that a minor merging, while producing only a small fraction $(\sim 2 \%)$ of new stars, is sufficient to disturb the morphology and the dynamics of a giant galaxy.

\section{Discussion}

\subsection{The origin of the $\mathrm{H} \alpha$ trails}

The high star formation associated with the galaxies in BIG, the high metal content of the extragalactic HII regions, and the unusual properties of CGCG97-125, are observed in typical compact groups and are strongly suggestive of tidal interaction between the group's members. Therefore it is certain that gravitational interactions are strongly influencing the evolutionary history of this group.

Are the multiple trails of ionized gas consistent with a tidal interaction scenario? One of the $\mathrm{H} \alpha$ trails apparently traces a perfect loop around CGCG97-120 (see Fig. 2). Yet this galaxy seems not to be associated with BIG, having a recessional velocity of $5635 \mathrm{~km} \mathrm{~s}^{-1}$, thus blue-shifted with respect to A1367 by approximately $800 \mathrm{~km} \mathrm{~s}^{-1}$. Is this a pure coincidence? The galaxy's morphology and kinematics are completely unperturbed, showing no signs of interaction (see Figs. 1 and 6). Moreover the scattering angle between a satellite galaxy and CGCG97-120 would be only 4-10 degrees, assuming a classical scattering model and an impact parameter of $\sim 10 \mathrm{kpc}$, too small to produce the observed loop. Thus we must assume that the association between CGCG97-120 and the $\mathrm{H} \alpha$ trails is a mere coincidence.

Whatever the role of CGCG97-120 is, it is indisputable that the geometry of the $\mathrm{H} \alpha$ trails is strongly suggestive of a 


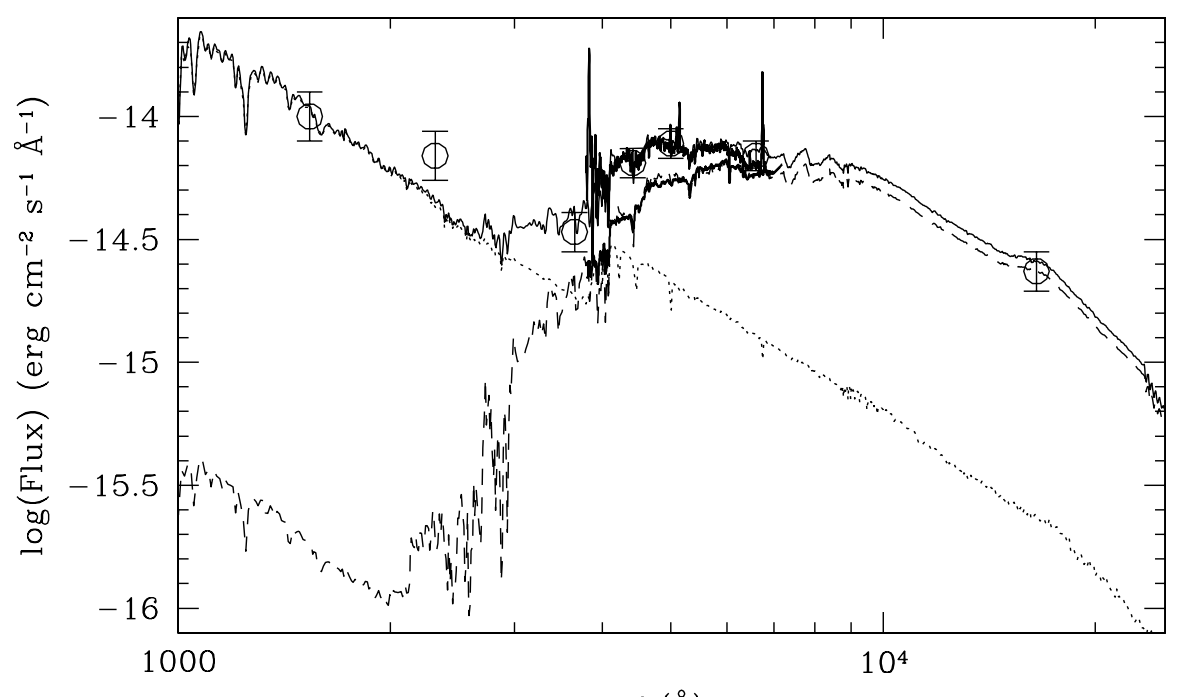

$\lambda(\AA)$

Fig. 12. The SED of CGCG97-125, corrected for internal extinction. Solid thick lines indicate the optical nuclear and drift-scan spectra. Circles indicate the photometric observations and their uncertainties. Best fitting models for the nuclear spectrum (dashed line) and for the starburst component (dotted line) are given. The solid thin line shows the resulting best fitting SED for CGCG97-125.

Rosetta orbit typical of the tidal disruption of a satellite galaxy. Contrary to other known examples of tidal streams (i.e. Ibata et al. 2001; Shang et al. 1998; Wehner \& Gallagher 2005), the features here observed show strong $\mathrm{H} \alpha$ emission. Tidal streams discovered in interacting systems (e.g. Shang et al. 1998; Forbes et al. 2003) are in fact detected only in continuum with no $\mathrm{H} \alpha$ emission, even if associated to strong starburst merging systems (Wehner \& Gallagher 2005). The case offered by BIG seems therefore unique among interacting systems as it is characterized by strong emission line trails.

Even if they have never been observed among other interacting systems, similar $\mathrm{H} \alpha$ trails have been recently discovered in the NW part of Abell 1367 itself. Gavazzi et al. (2001a) discovered two low surface brightness $\mathrm{H} \alpha$ cometary tails, with a projected length of $75 \mathrm{kpc}$, associated with two star forming systems: CGCG97-073 and CGCG97-079. The morphology of these tails (whose typical size and gas densities are similar to the trails observed in BIG) suggests that galaxies in the NW group are experiencing ram pressure due to their high velocity motion through the IGM (Gavazzi et al. 2001a). The difference between the case of CGCG97-079 and CGCG97-073 and BIG is that the former are isolated systems infalling into the cluster, while galaxies in BIG are infalling within a compact group, where gravitational interactions are not negligible. For these reasons, the unique features observed in BIG lead to the supposition that it is not only tidal interaction which can produce the $\mathrm{H} \alpha$ trails but that probably the mutual influence of tidal and ram pressure can also explain the properties of the $\mathrm{H} \alpha$ trails, unless we are dealing with a unique example of tidal trail with associated ionized gas, on scales exceeding $150 \mathrm{kpc}$. Since BIG is infalling at $1700 \mathrm{~km} \mathrm{~s}^{-1}$ into the center of Abell 1367, ram pressure is likely playing a non-negligible role on the evolution of this group.

This scenario seems supported by the new HI observations obtained at Arecibo. The HI profile indicates the presence of diffused neutral gas probably associated with the $\mathrm{H} \alpha$ trail. This diffuse component show a recessional velocity in the range between 7500 and $8000 \mathrm{~km} \mathrm{~s}^{-1}$ (systematically lower than the typical velocity of the star forming systems), as if the gas was ram-pressure stripped from galaxies infalling at $1700 \mathrm{~km} \mathrm{~s}^{-1}$ and experienced a progressive velocity decrease due to the friction with the cluster IGM $\left(V \sim 6500 \mathrm{~km} \mathrm{~s}^{-1}\right)$. In this perspective the properties of the HI diffused component in BIG are not too different from the ones observed in the extended HI plume recently discovered in Virgo and interpreted by Oosterloo \& van Gorkom (2005) as due to ram pressure stripping from NGC 4388. The $\mathrm{H} \alpha$ trails might therefore be suggesting that both mechanisms (i.e. tidal interactions among the group members and ram pressure with the cluster IGM) are taking place at once, as already observed in the Virgo cluster (Vollmer 2003; Vollmer et al. 2005a,b). Deeper observations and numerical simulations are however mandatory to confirm the role of ram pressure stripping on shaping the properties of this group.

\subsection{The ionization source of the trails}

The large extent of the $\mathrm{H} \alpha$ trails and their associated neutral component indicates that stripped gas can survive from $100 \mathrm{Myr}$ up to $1.5 \mathrm{Gyr}$ (see Sect. 3.1). This represents a problem for the diffused HI in BIG as well as in other known extended HI components in clusters (i.e. Oosterloo \& van Gorkom 2005) because HI evaporation by the hot IGM should occur in few $10^{7} \mathrm{yr}$ (Cowie \& McKee 1977). One possibility is that HI evaporation is in fact less effective because the gas is shielded by tangled magnetic fields (Cowie et al. 1981; Vollmer et al. 2001).

Furthermore the plasma density derived in Sect. 3.1 implies an exceedingly short recombination time for the ionized component along the trails of BIG: $\tau_{\mathrm{r}}=1 / N_{\mathrm{e}} \alpha_{\mathrm{a}} \sim 2-7 \mathrm{Myr}$, where $\alpha_{\mathrm{a}}=4.2 \times 10^{-13} \mathrm{~cm}^{3} \mathrm{~s}^{-1}$ (Osterbrock 1989). Can this exceedingly short recombination time of few Myr be reconciled with an age of the phenomenon between some 100 Myr and 1.5 Gyr? We need a mechanism to keep the gas ionized for such a long time. In spiral galaxies, if the HI column density is above a few times $10^{20} \mathrm{~cm}^{-2}$, star formation almost invariably occurs (Boissier et al. 2003). The mean column density in the trail is consistent with this value, suggesting that the star formation threshold might locally be exceeded. Hence, star formation could in principle provide the source of ionization in the trails. However we have no evidence of star formation along the trails and the dynamical picture of BIG is consistent with the idea that at least part of the gas has been stripped ionized from the galaxies. 

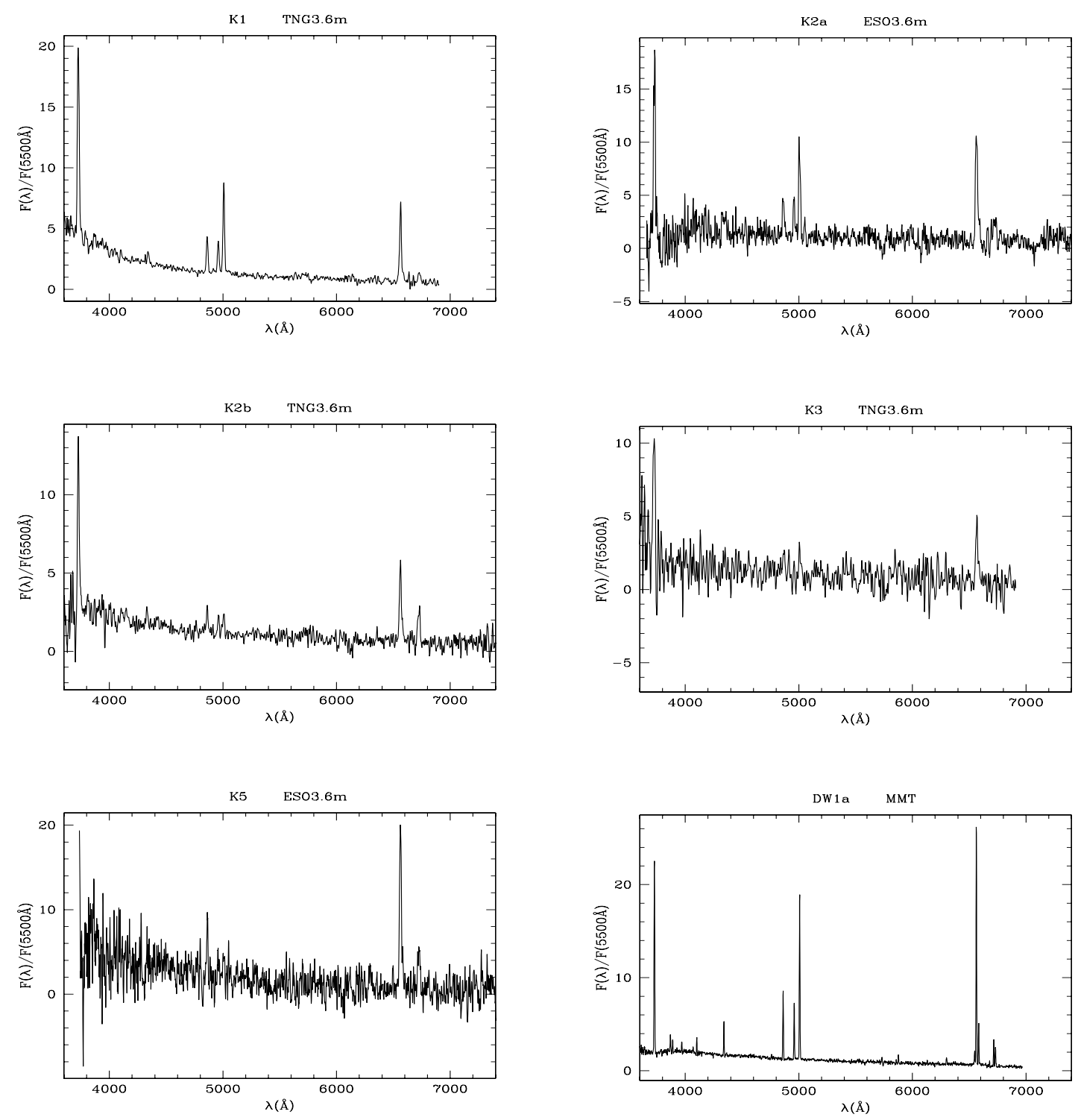

Fig. 13. The observed, smoothed (step 3), one dimensional spectra. The object identification and telescope are labeled on each panel.

A source of ionization for the trails could be provided by UV photons escaping from the star-bursting regions within the main group's galaxies, as observed in other starburst systems (i.e. Morse et al. 1998; Devine \& Bally 1999; Hameed et al. 2001). However the UV continuum emitted by the two bright galaxies CGCG97125 and CGCG97-114 could only explain the presence of diffuse ionized gas observed in their surroundings $(<10-20 \mathrm{kpc}$ from the center), but it is unlikely responsible for the ionization of the $\mathrm{H} \alpha$ trails (see also Veilleux et al. 2003).

Another possibility is that the gas is ionized by the propagation of shock waves (Jog \& Solomon 1992; Barnes 2004) produced during the galaxies collisions and/or the high velocity infall into the hot intracluster medium. Deep spectroscopic observations are necessary to test this possibility. Finally, we must consider the effect of the bremsstrahlung radiation by the ICM on the stripped gas. Following Vollmer et al. (2001) and Maloney et al. (1996) the X-ray ionizing photon flux $\left(\phi_{\mathrm{i}}\right)$ is:

$\phi_{\mathrm{i}}=\frac{\ln (0.1 / 0.0136)}{1.6 \times 10^{-9}} \frac{F_{\mathrm{X}}}{1.5}=8.3 \times 10^{8} F_{\mathrm{X}}$ photons $\mathrm{cm}^{-2} \mathrm{~s}^{-1}$

where $F_{\mathrm{X}}$ is the X-ray flux. Assuming a total cluster X-ray luminosity of $4 \times 10^{43} \mathrm{erg} \mathrm{cm}^{-2}$ (Donnelly et al. 1998) the X-ray flux at a projected distance of $\sim 125 \mathrm{kpc}$ from the $X$-ray center (where BIG is observed) is $\sim 2.5 \times 10^{-6} \mathrm{erg} \mathrm{cm}^{-2} \mathrm{~s}^{-1}$ and $\phi_{\mathrm{i}}$ results $\sim 2.1 \times 10^{3}$ photons $\mathrm{cm}^{-2} \mathrm{~s}^{-1}$. In equilibrium this gives rise to an ionized column density $N_{\mathrm{e}}=\phi_{\mathrm{i}} / \alpha_{\mathrm{a}} n_{\mathrm{e}}$. Using $n_{\mathrm{e}}=10^{-2} \mathrm{~cm}^{-3}$ we obtain $N_{\mathrm{e}} \sim 5 \times 10^{20} \mathrm{~cm}^{-2}$, consistent with value measured in the ionized tails (see Sect. 3.1). This simple calculation shows that the stripped gas can be kept ionized by the X-ray photons. A deep spectrum of the trails will help us solving this riddle.

\subsection{The Blue Infalling Group: an unique example among compact groups?}

The features observed in BIG are indicative that tidal forces and ram pressure are mutually shaping the evolution of this system. In fact BIG appears as an unique example of a compact group infalling into the core of a cluster of galaxies.

Is BIG really so different from "normal compact groups"?

In order to answer to this question, let us compare BIG with the prototype of compact groups: the Stephan's Quintet (HCG92, hereafter SQ). This group is composed of four interacting galaxies and, lying at approximately the same distance of BIG $\left(\sim 92 h_{70}^{-1} \mathrm{Mpc}\right)$, is ideal for a comparative study. 

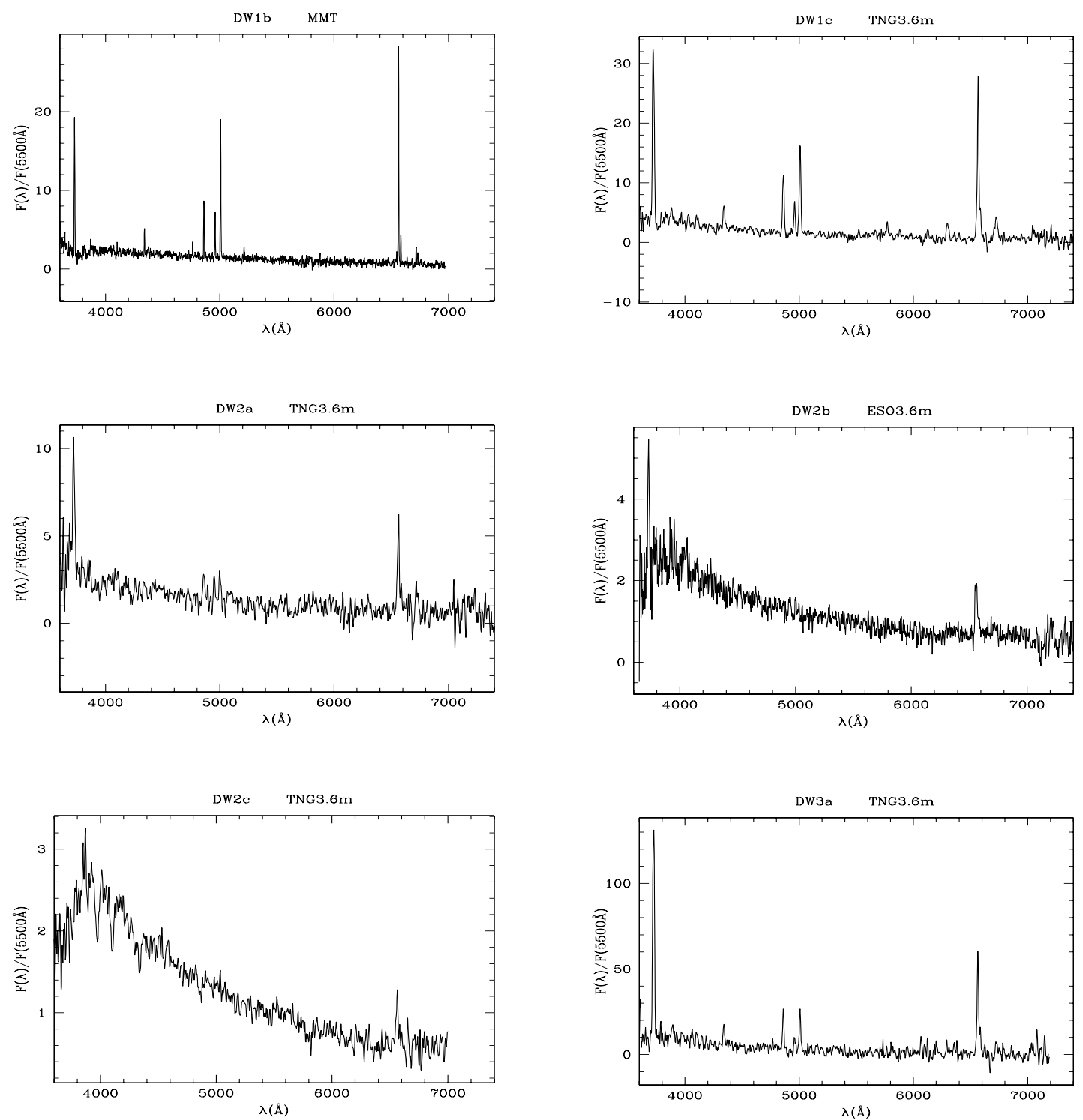

Fig. 13. continued.

Both groups show a high number of bright, metal rich tidal dwarfs/extragalactic HII regions (Iglesias-Páramo \& Vílchez 2001; Mendes de Oliveira et al. 2001, 2004) and diffuse HI components (with a total HI mass of $M \sim 10^{9}-10^{10} M_{\odot}$ ), suggesting that tidal interactions are perturbing the groups' members. While in the SQ we observe two tidal tails with a mean surface brightness of $\mu_{V}=24.4$ and $26 \mathrm{mag} \operatorname{arcsec}^{-2}$ (Sulentic et al. 2001) respectively, a stellar trail with a typical surface brightness of $\mu_{r^{\prime}} \sim 26 \mathrm{mag} \operatorname{arcsec}^{-2}$ and diffuse intra-group light $\left(25.2 \leq \mu_{r^{\prime}} \leq 26.6 \mathrm{mag} \operatorname{arcsec}^{-2}\right)$ are observed in BIG. However BIG shows multiple $\mathrm{H} \alpha$ trails and streams with a total extension of $150 \mathrm{kpc}$, features not observed in the SQ (Xu et al. 1999; Sulentic et al. 2001). The neutral hydrogen component associated with these streams has a recessional velocity in the range between 7500 and $8000 \mathrm{~km} \mathrm{~s}^{-1}$, and is systematically blue-shifted with respect to the mean velocity of the star forming systems. On the contrary, in the SQ the diffuse HI lies at the same velocity of the star forming regions (Williams et al. 2002). As discussed in Sect. 4.1 the properties of the diffuse $\mathrm{H} \alpha$ emission and its associated HI component, not observed in the SQ, are consistent with a ram pressure stripping scenario, supporting the idea that the differences between BIG and SQ are due to the interaction of BIG with the cluster IGM.

Finally, a galaxy with recessional velocity significantly different from the mean group's velocity is observed in both BIG and SQ. In BIG, CGCG97-120 has a recessional velocity $\sim 2500 \mathrm{~km} \mathrm{~s}^{-1}$ lower than the group's velocity, while in the SQ NGC $7318 \mathrm{~b}$ is blue-shifted by $\sim 900 \mathrm{~km} \mathrm{~s}^{-1}$ with respect to other members. Multiwavelength observations of SQ (Sulentic et al. 2001; Xu et al. 1999, 2003, 2005) have shown that NGC 7318b is currently colliding with the intra group medium, triggering a large-scale shock and a burst of star formation. On the contrary no shocks are observed in BIG and, excluding the H $\alpha$ loop around CGCG97-120, no observational evidences of an interaction between this galaxy and BIG are detected. Looking at Fig. 2, it is difficult to believe that the association between CGCG97-120 and the $\mathrm{H} \alpha$ trails is a mere coincidence; however without independent observations it is impossible to determine whether or not we are witnessing an extremely high velocity interaction as in the SQ.

\section{Conclusions}

The considerable observational material presented in this paper allows us to propose an evolutionary history for BIG over the last 1-2 Gyr. Originally BIG was a normal 

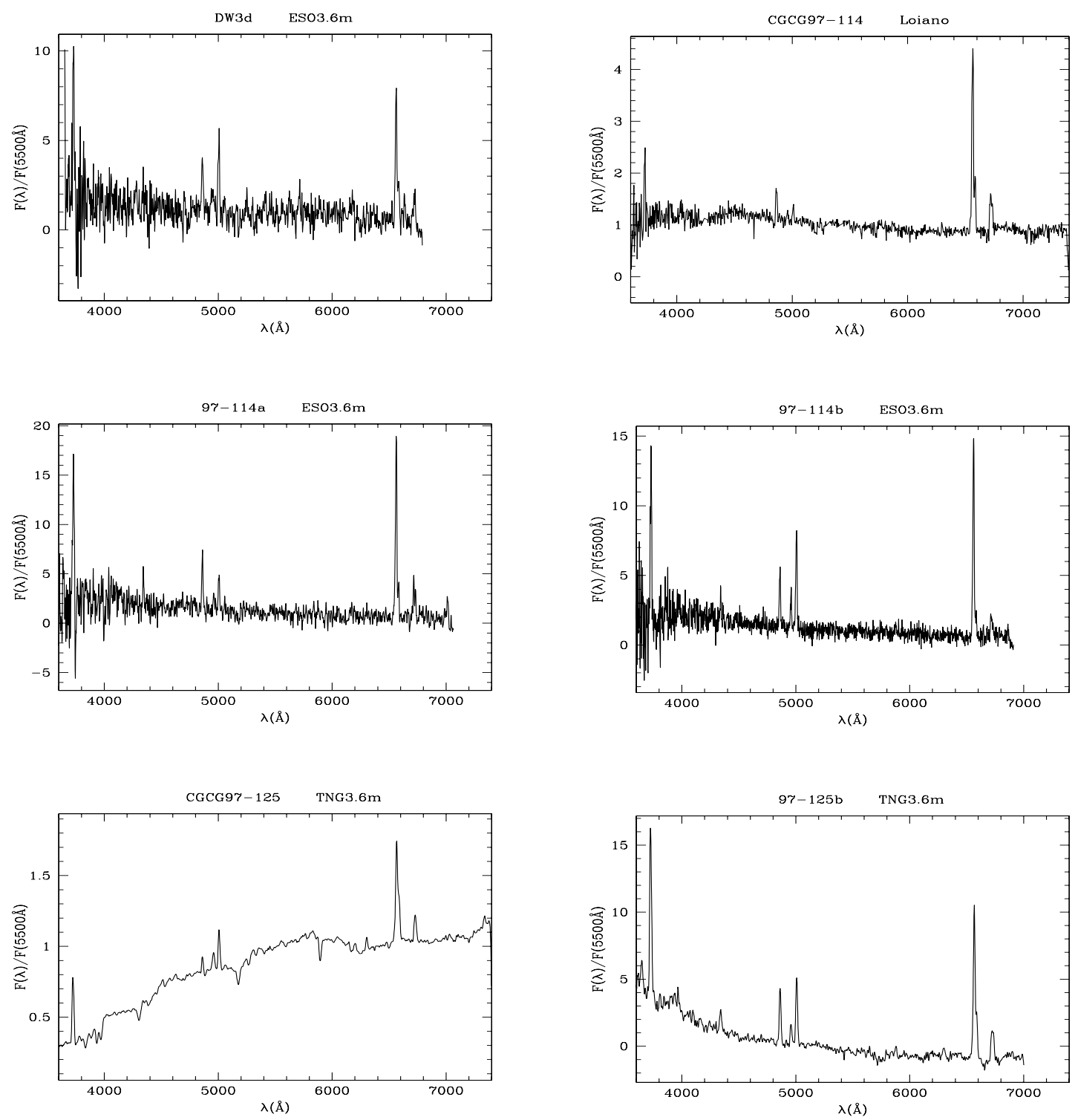

Fig. 13. continued.

compact group of galaxies with a typical velocity dispersion of $\sim 150-200 \mathrm{~km} \mathrm{~s}^{-1}$, composed of at least three galaxies: a massive evolved early type galaxy (CGCG97-125), a less massive late type spiral (CGCG97-114) and a gas rich dwarf galaxy (the satellite presently merged with CGCG97-125) with a stellar mass $\sim 10^{9} M_{\odot}$. Lying in the outskirts of Abell 1367 the group has been attracted by the cluster potential and started its infall into the cluster core at a speed of $\sim 1700 \mathrm{~km} \mathrm{~s}^{-1}$. During the journey, its galaxies were perturbed by mutual gravitational interactions, as is often observed in compact groups, resulting in star and gas stripping, tidal tails (K2), extragalactic compact HII regions (K5 and K1) and tidal dwarfs (DW1, DW2 and DW3). Finally the gas rich satellite merged into CGCG97-125 producing stellar shells and a burst of star formation. In the meanwhile tidal interactions also weakened the potential wells of the group galaxies, making it easier for ram pressure to strip the galaxies' ISM, producing the unique $\mathrm{H} \alpha$ trails.

Galaxy clusters grow by accreting small groups of galaxies falling in along large scale filaments. The group on which we focused the present investigation might represent an unique laboratory as it reproduces, at the present epoch, the physical conditions that were likely to exist in clusters under formation. What can we learn about galaxy/cluster evolution by studying

BIG? The brightest system in BIG, CGCG97-125, appears as a S0 galaxy that has recently $(\sim 1.5 \mathrm{Gyr})$ experienced a minor merging event and a consequent burst of star formation, implying that S0 galaxies can form outside clusters and subsequently join into them. Groups are the natural sites where gravitational interactions can produce such transformation of normal spirals into S0s, a mechanism that has been named preprocessing.

Tidal interactions within the group's members cannot only induce morphological transformations in galaxies, but also can create new systems from stripped material, as shown by the high number of metal rich star forming dwarfs/extragalactic HII region detected in BIG. What is the future of these stripped systems? If they are dynamically bounded, they might represents the progenitors of the population of cluster dwarf galaxies. Otherwise they will disperse their stars into the intracluster medium contributing to the Abell 1367's intracluster light. The unique $\mathrm{H} \alpha$ trails observed in BIG suggest that a considerable amount of the ICM enrichment might derive from infalling groups, as opposed to winds from elliptical galaxies, which are commonly accepted as the major sources of pollution (e.g. De Lucia et al. 2004; Madau et al. 2001; Mori et al. 2002). Recent simulations have in fact shown that more of the $10 \%$ of the intracluster medium originated from the stripping of 
gas-rich systems by ram pressure (Domainko et al. 2005). Two more galaxies with associated $\mathrm{H} \alpha$ trails are indeed observed in the NW part of Abell 1367 itself (Gavazzi et al. 2001a) emphasizing that a great deal of transformation is taking place in a dynamically young cluster even at the present cosmological epoch.

Acknowledgements. We thank the referee, P. A. Duc, for his useful comments which helped us to improve and strengthen the paper. We wish to thank Samuel Boissier for useful discussions. This research has made use of the GOLDMine Database, operated by the Universitá degli Studi di Milano-Bicocca.

\section{References}

Barnes, J. E. 2004, MNRAS, 350, 798

Boissier, S., Prantzos, N., Boselli, A., \& Gavazzi, G. 2003, MNRAS, 346, 1215 Boselli, A., \& Gavazzi, G. 2002, A\&A, 386, 124

Boselli, A., Gavazzi, G., Donas, J., \& Scodeggio, M. 2001, AJ, 121, 753

Boselli, A., Iglesias-Páramo, J., Vúlchez, J. M., \& Gavazzi, G. 2002, A\&A, 386, 134

Boselli, A., Gavazzi, G., \& Sanvito, G. 2003, A\&A, 402, 37

Boselli, A., Boissier, S., Cortese, L., et al. 2005, ApJ, 623, L13

Bravo-Alfaro, H., Cayatte, V., van Gorkom, J. H., \& Balkowski, C. 2000, AJ, 119,580

Bresolin, F., \& Kennicutt, R. C. 1997, AJ, 113, 975

Bruzual, G., \& Charlot, S. 2003, MNRAS, 344, 1000

Cayatte, V., van Gorkom, J. H., Balkowski, C., \& Kotanyi, C. 1990, AJ, 100, 604

Cortese, L., Gavazzi, G., Boselli, A., Iglesias-Paramo, J., \& Carrasco, L. 2004, A\&A, 425, 429

Cortese, L., Boselli, A., Gavazzi, G., et al. 2005, ApJ, 623, L17

Cortese, L., Boselli, A., Buat, V., et al. 2006, ApJ, 637, 242

Cowie, L. L., \& McKee, C. F. 1977, ApJ, 211, 135

Cowie, L. L., McKee, C. F., \& Ostriker, J. P. 1981, ApJ, 247, 908

De Lucia, G., Kauffmann, G., \& White, S. D. M. 2004, MNRAS, 349, 1101

Devine, D., \& Bally, J. 1999, ApJ, 510, 197

Domainko, W., Mair, M., Kapferer, W., et al. 2005 [arXiv: astro-ph/0507605]

Donnelly, R. H., Markevitch, M., Forman, W., et al. 1998, ApJ, 500, 138

Donnelly, R. H., Forman, W., Jones, C., et al. 2001, ApJ, 562, 254

Dressler, A. 1980, ApJ, 236, 351

Dressler, A. 2004, in Clusters of Galaxies: Probes of Cosmological Structure and Galaxy Evolution, 207

Duc, P.-A., \& Mirabel, I. F. 1998, A\&A, 333, 813

Duc, P.-A., \& Mirabel, I. F. 1999, in Galaxy Interactions at Low and High Redshift, IAU Symp., 186, 61

Duc, P.-A., Brinks, E., Springel, V., et al. 2000, AJ, 120, 1238

Dutil, Y., \& Roy, J. 1999, ApJ, 516, 62

Ferrari, C., Maurogordato, S., Cappi, A., \& Benoist, C. 2003, A\&A, 399, 813

Forbes, D. A., Beasley, M. A., Bekki, K., Brodie, J. P., \& Strader, J. 2003, Science, 301, 1217

Franzetti, P. 2005, Ph.D. Thesis, Universitá degli Studi di Milano-Bicocca

Fujita, Y. 2004, PASJ, 56, 29

Gavazzi, G., Catinella, B., Carrasco, L., Boselli, A., \& Contursi, A. 1998, AJ, 115,1745

Gavazzi, G., Boselli, A., Scodeggio, M., Pierini, D., \& Belsole, E. 1999, MNRAS, 304, 595

Gavazzi, G., Boselli, A., Mayer, L., et al. 2001a, ApJ, 563, L23

Gavazzi, G., Marcelin, M., Boselli, A., et al. 2001b, A\&A, 377, 745

Gavazzi, G., Bonfanti, C., Sanvito, G., Boselli, A., \& Scodeggio, M. 2002a, ApJ, 576,135

Gavazzi, G., Boselli, A., Pedotti, P., Gallazzi, A., \& Carrasco, L. 2002b, A\&A, 386,114

Gavazzi, G., Boselli, A., Pedotti, P., Gallazzi, A., \& Carrasco, L. 2002c, A\&A, 396,449

Gavazzi, G., Boselli, A., Donati, A., Franzetti, P., \& Scodeggio, M. 2003a, A\&A, 400, 451

Gavazzi, G., Cortese, L., Boselli, A., et al. 2003b, ApJ, 597, 210

Gavazzi, G., Zaccardo, A., Sanvito, G., Boselli, A., \& Bonfanti, C. 2004, A\&A, 417,499

Gavazzi, G., Boselli, A., Cortese, L., et al. 2006, A\&A, in press
Gottlöber, S., Klypin, A., \& Kravtsov, A. V. 2001, ApJ, 546, 223

Gunn, J. E., \& Gott, J. R. I. 1972, ApJ, 176, 1

Hameed, S., Blank, D. L., Young, L. M., \& Devereux, N. 2001, ApJ, 546, L97

Haynes, M. P., Jore, K. P., Barrett, E. A., Broeils, A. H., \& Murray, B. M. 2000, AJ, 120, 703

Ibata, R., Irwin, M., Lewis, G., Ferguson, A. M. N., \& Tanvir, N. 2001, Nature, 412, 49

Iglesias-Páramo, J., Boselli, A., Cortese, L., Vílchez, J. M., \& Gavazzi, G. 2002, A\&A, 384, 383

Iglesias-Páramo, J., \& Vílchez, J. M. 2001, ApJ, 550, 204

Jog, C. J., \& Solomon, P. M. 1992, ApJ, 387, 152

Jore, K. P., Broeils, A. H., \& Haynes, M. P. 1996, AJ, 112, 438

Kauffmann, G., Heckman, T. M., White, S. D. M., et al. 2003, MNRAS, 341, 33

Kennicutt, R. C., Keel, W. C., \& Blaha, C. A. 1989, AJ, 97, 1022

Kewley, L. J., \& Dopita, M. A. 2002, ApJS, 142, 35

Kobulnicky, H. A., Kennicutt, R. C., \& Pizagno, J. L. 1999, ApJ, 514, 544

Kodama, T., Smail, I., Nakata, F., Okamura, S., \& Bower, R. G. 2001, ApJ, 562, L9

Kojima, M., \& Noguchi, M. 1997, ApJ, 481, 132

Lequeux, J., Peimbert, M., Rayo, J. F., Serrano, A., \& Torres-Peimbert, S. 1979, A\&A, 80, 155

Madau, P., Ferrara, A., \& Rees, M. J. 2001, ApJ, 555, 92

Maloney, P. R., Hollenbach, D. J., \& Tielens, A. G. G. M. 1996, ApJ, 466, 561

McGaugh, S. S. 1991, ApJ, 380, 140

Mendes de Oliveira, C., Plana, H., Amram, P., Balkowski, C., \& Bolte, M. 2001, AJ, 121, 2524

Mendes de Oliveira, C., Cypriano, E. S., Sodré, L., \& Balkowski, C. 2004, ApJ, 605, L17

Mihos, J. C. 2004, in Clusters of Galaxies: Probes of Cosmological Structure and Galaxy Evolution, 278

Moore, B., Katz, N., Lake, G., Dressler, A., \& Oemler, A. 1996, Nature, 379, 613

Mori, M., Ferrara, A., \& Madau, P. 2002, ApJ, 571, 40

Morse, J. A., Cecil, G., Wilson, A. S., \& Tsvetanov, Z. I. 1998, ApJ, 505, 159

Mulchaey, J. S. 2000, ARA\&A, 38, 289

O'Neil, K. 2004, AJ, 128, 2080

Oosterloo, T., \& van Gorkom, J. 2005, A\&A, 437, L19

Osterbrock, D. E. 1989, Astrophysics of gaseous nebulae and active galactic nuclei (Research supported by the University of California, John Simon Guggenheim Memorial Foundation, University of Minnesota, et al. Mill Valley, CA, University Science Books), 422

Poggianti, B. M., \& Barbaro, G. 1997, A\&A, 325, 1025

Poggianti, B. M., Bridges, T. J., Mobasher, B., et al. 2001, ApJ, 562, 689

Poggianti, B. M., Smail, I., Dressler, A., et al. 1999, ApJ, 518, 576

Rubin, V. C., Waterman, A. H., \& Kenney, J. D. P. 1999, AJ, 118, 236

Sakai, S., Mould, J. R., Hughes, S. M. G., et al. 2000, ApJ, 529, 698

Sakai, S., Kennicutt, R. C., van der Hulst, J. M., \& Moss, C. 2002, ApJ, 578, 842

Salpeter, E. E. 1955, ApJ, 121, 161

Shang, Z., Brinks, E., Zheng, Z., et al. 1998, ApJ, 504, L23

Skillman, E. D., Kennicutt, R. C., \& Hodge, P. W. 1989, ApJ, 347, 875

Smail, I., Ellis, R. S., Dressler, A., et al. 1997, ApJ, 479, 70

Sulentic, J. W., Rosado, M., Dultzin-Hacyan, D., et al. 2001, AJ, 122, 2993

Sun, M., \& Murray, S. S. 2002, ApJ, 576, 708

Treu, T., Ellis, R. S., Kneib, J.-P., et al. 2003, ApJ, 591, 53

van Zee, L., Salzer, J. J., Haynes, M. P., O’Donoghue, A. A., \& Balonek, T. J. 1998, AJ, 116, 2805

Veilleux, S., Shopbell, P. L., Rupke, D. S., Bland-Hawthorn, J., \& Cecil, G. 2003, AJ, 126, 2185

Vollmer, B. 2003, A\&A, 398, 525

Vollmer, B., Cayatte, V., Balkowski, C., \& Duschl, W. J. 2001, ApJ, 561, 708

Vollmer, B., Braine, J., Combes, F., \& Sofue, Y. 2005a, ArXiv Astrophysics eprints

Vollmer, B., Huchtmeier, W., \& van Driel, W. 2005b, A\&A, 439, 921

Wehner, E. H., \& Gallagher, J. S. 2005, ApJ, 618, L21

Williams, B. A., Yun, M. S., \& Verdes-Montenegro, L. 2002, AJ, 123, 2417

Xu, C., Sulentic, J. W., \& Tuffs, R. 1999, ApJ, 512, 178

Xu, C. K., Lu, N., Condon, J. J., Dopita, M., \& Tuffs, R. J. 2003, ApJ, 595, 665

Xu, C. K., Iglesias-Páramo, J., Burgarella, D., et al. 2005, ApJ, 619, L95

Zaritsky, D., Kennicutt, R. C., \& Huchra, J. P. 1994, ApJ, 420, 87

Zwicky, F., Herzog, E., \& Wild, P. 1961, Catalogue of galaxies and of clusters of galaxies (Pasadena: California Institute of Technology, CIT) 